\title{
THE EQUIVARIANT COHOMOLOGY THEORY OF TWISTED GENERALIZED COMPLEX MANIFOLDS
}

\author{
YI LIN
}

\begin{abstract}
It has been shown recently by Kapustin and Tomasiello that the mathematical notion of Hamiltonian actions on twisted generalized Kähler manifolds is in perfect agreement with the physical notion of general $(2,2)$ gauged sigma models with three-form fluxes. In this article, we study the twisted equivariant cohomology theory of Hamiltonian actions on $\mathrm{H}$-twisted generalized complex manifolds. If the manifold satisfies the $\bar{\partial} \partial$-lemma, we establish the equivariant formality theorem. If in addition, the manifold satisfies the generalized Kähler condition, we prove the Kirwan injectivity in this setting. We then consider the Hamiltonian action of a torus on an $\mathrm{H}$-twisted generalized Calabi-Yau manifold and extend to this case the Duistermaat-Heckman theorem for the pushforward measure.

As a side result, we show in this paper that the generalized Kähler quotient of a generalized Kähler vector space can never have a (cohomologically) non-trivial twisting. This gives a negative answer to a question asked by physicists whether one can construct $(2,2)$ gauged linear sigma models with non-trivial fluxes.
\end{abstract}

\section{INTRODUCTION}

Generalized complex geometry was initiated by Hitchin [H02] and further developed by Gualtieri in [Gua03]. It provides a unifying framework for both symplectic geometry and complex geometry, and turns out to be a useful geometric language understanding various aspects of string theory. Generalized Kähler geometry, the generalized complex version of Kähler geometry, was introduced by Gualtieri, who also shows that it is equivalent to the bi-Hermitian geometry which was first discovered by Gates, Hull and Rocek [GHR84] from the study of general $(2,2)$ supersymmetric sigma models.

In [LT05], Tolman and the author proposed a definition of a Hamiltonian action and a moment map in both generalized complex geometry and generalized Kähler geometry. Recently, it has been shown by Kapustin and Tomasiello [KT06] that the mathematical notion of Hamiltonian actions on generalized Kähler manifolds introduced in [LT05] is in perfect agreement with the expectations from renormalization group flow, and corresponds

Date: November 9, 2018. 
exactly to the physical notion of general $(2,2)$ gauged sigma models with three-form fluxes.

In this paper, we study the equivariant cohomology theory of Hamiltonian actions on twisted generalized complex and Kähler manifolds. To better explain the ideas involved in this work, let us first state an equivalent definition of Hamiltonian actions on generalized complex manifolds. Suppose a compact Lie group $\mathrm{G}$ with Lie algebra $\mathfrak{g}$ acts on an H-twisted generalized complex manifold $(M, \mathcal{J})$, where $H$ is a closed three form on $M$. The action is said to be Hamiltonian if there exists an equivariant smooth function $\mu: M \rightarrow \mathfrak{g}^{*}$, called the generalized moment map, a $\mathfrak{g}^{*}$-valued one form $\alpha \in \Omega^{1}\left(M, \mathfrak{g}^{*}\right)$, called the moment one form, such that $\mathrm{H}+\alpha$ is equivariantly closed (in the usual Cartan model) and such that the following diagram commutes:

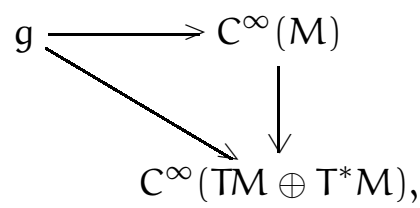

where the horizontal map is given by $\xi \mapsto f^{\xi}$, the vertical map is given by $f \mapsto-\mathcal{J} d f$, and the diagonal map is given by $\xi \mapsto \xi_{M}+\alpha^{\xi}$. Therefore, morally speaking, the Hamiltonian action of a compact Lie group $G$ not only defines an action on the manifold $M$, but also defines an extended action on the exact Courant algebroid $\mathrm{TM} \oplus \mathrm{T}^{*} \mathrm{M}$. 1

The equivariant cohomology theory has been an important topic in the study of group actions on manifolds. However, the usual equivariant cohomology theory encodes only the cohomological information of group actions on manifolds, and does not give us any information on the extended group actions on the exact Courant algebroid $\mathrm{TM} \oplus \mathrm{T}^{*} \mathrm{M}$. So when considering the consequence of the $\bar{\partial} \partial$-lemma for Hamiltonian actions on generalized complex manifolds, the author [Lin06] was forced to introduce two extensions of the usual equivariant de Rham cohomology theory, called the generalized equivariant cohomology and the generalized equivariant Dolbeault cohomology, to encode the information of the extended actions on $\mathrm{TM} \oplus \mathrm{T}^{*} \mathrm{M}$.

In this paper, we explain that the generalized equivariant cohomology introduced in [Lin06] is actually the usual equivariant cohomology twisted by the equivariantly closed three form $\mathrm{H}+\alpha$. We note that the equivariant cohomology theory of general extended group actions on exact Courant

\footnotetext{
${ }^{1}$ Indeed, the elements of extended group actions on exact Courant algebroids has been developed in [Hu05], and, more generally, in [BCG05].
} 
algebroids has been developed by $\mathrm{Hu}$ and Uribe [HuUribe06]. 2 In particular, $\mathrm{Hu}$ and Uribe proved that the twisted equivariant cohomology has the usual expected properties of an equivariant cohomology theory such as functoriality, Mayer-Vietoris lemma, Thom isomorphism, localization theorem, etc.

In this context, the equivariant $\bar{\partial} \partial$-lemma obtained in [Lin06] can be best understood in the framework of twisted equivariant cohomology theory. Let us consider the Hamiltonian action of a compact Lie group on an $\mathrm{H}$ twisted generalized complex manifold satisfying the $\bar{\partial} \partial-$ lemma. As an immediate consequence of the equivariant $\bar{\partial} \partial$-lemma, we obtain the equivariant formality of the twisted equivariant cohomology $\mathrm{H}_{\mathrm{G}}(\mathrm{M}, \mathrm{H}+\alpha)$. Now assume the compact Lie group is a torus $\mathrm{T}$, and assume that the manifold satisfies the generalized Kähler condition, we establish the Kirwan injectivity theorem for the twisted equivariant cohomology $\mathrm{H}_{T}(M, H+\alpha)$.

It is an interesting question that if we can understand the cohomology of the generalized Kähler quotients from the cohomology of the Hamiltonian generalized Kähler manifold upstairs. We believe that the equivariant formality theorem and Kirwan injectivity theorem we established in this paper is useful for answering such a question. As a side result, we show that the generalized Kähler quotient of a generalized Kähler vector space can never have a (cohomologically) non-trivial twisting. This gives a negative answer to a question asked by physicists whether one can construct gauged linear sigma models with non-trivial fluxes.

As an application of the twisted equivariant cohomology, we investigate the Hamiltonian action of a torus $\mathrm{T}$ on an $\mathrm{H}$-twisted generalized Calabi-Yau manifold $(M, \rho)$, where $\rho$ is an $\mathrm{H}$-twisted generalized Calabi-Yau structure, i.e., a nowhere vanishing section of the canonical line bundle of the generalized complex manifold $M$ such that $\mathrm{d} \rho=\mathrm{H} \wedge \rho$ for the closed three form $\mathrm{H}$.

Indeed, the Hamiltonian action on generalized Calabi-Yau manifolds has been studied in the very recent works of Nitta, [NY06], [NY07]. Under the assumption that both the twisting three form $\mathrm{H}$ and the moment one form $\alpha$ are zeroes, Nitta showed that the generalized Calabi-Yau structure descends to the generalized complex quotient. More interestingly, he observed that the Mukai pairing $(\rho, \bar{\rho})$ gives rise to a volume form and so a measure on the generalized Calabi-Yau manifold; and if the type of the generalized Calabi-Yau structure is constant, then the push-froward measure via the generalized moment map is absolutely continuous and varies as a

\footnotetext{
${ }^{2}$ We would also like to mention that the equivariant Lie algebroid cohomology, as introduced in [BCRR05], has been first studied in [BCG05, Sec. 5] in the context of generalized complex geometry.
} 
polynomial in the connected component of the regular values of the generalized moment map. This has thus generalized the usual DuistermaatHeckman theorem to the setting of generalized Calabi-Yau manifolds. 3

However, the assumptions that both the twisting three form $\mathrm{H}$ and the moment one form $\alpha$ equal zero are not natural when one studies group actions on generalized complex manifolds. Indeed, even when the generalized complex manifold is untwisted, the moment one form may come up very naturally [LT05]. In this article, we show that neither one of these two assumptions made in [NY07] are necessary, establishing the DuistermaatHeckman theorem in the setting of twisted generalized Calabi-Yau geometry in full generality. To achieve this we need to introduce the equivariant differential forms with coefficients in the ring of formal power series, which we discuss in the appendix.

Along the same line, we explain that the notion of Hamiltonian action on generalized Calabi-Yau manifolds generalizes that of Hamiltonian action on symplectic manifolds. In addition, we give concrete examples of Hamiltonian action on (non-symplectic) generalized Calabi-Yau manifolds and calculate the density function of the push-forward measure via the generalized moment map explicitly.

Finally we would like to mention that there are four other groups who have worked independently on the reduction and quotient construction in generalized geometry: Bursztyn, Cavalcanti and Gualtieri [BCG05], S. $\mathrm{Hu}[\mathrm{Hu} 05]$, P. Xu and M. Stiénon [SX05], Vaisman, I. [Va05]. In particular, the connection between the work of Bursztyn, Cavalcanti and Gualtieri and the gauged sigma model in physics is explained in [BCG05, Thm. 2.13].

This paper is organized as follows. Section 2 gives a quick review of some foundational aspects of twisted cohomology and twisted equivariant cohomology. Section 3 reviews elements of generalized complex geometry. Section 4 establishes the equivariant formality and Kirwan injectivity theorems for the twisted equivariant cohomology of Hamiltonian actions on twisted generalized Kähler manifolds. Section 5 proves that the generalized Kähler quotients of generalized vector spaces can never have a nontrivial twisting. Section 6 establishes the Duistermaat-Heckman theorem in the setting of twisted generalized Calabi-Yau manifolds in full generality. Section 7 presents concrete examples of Hamiltonian action on generalized Calabi-Yau manifolds which are not symplectic, and computes the density function of the push-forward measure explicitly. Appendix A discusses the equivariant differential forms with coefficients in the ring of formal power series.

Acknowledgement: I would like to thank Tom Baird, Kentaro Hori, Lisa Jeffrey, Anton kapustin, Yael Karshon, Eckhard Meinrenken, Reyer Sjamaar, and Allessandro Tomasiello for useful conversations. And I would

\footnotetext{
${ }^{3}$ However, it seems that Nitta's proof [NY07] has a small gap. Please see Remark6.9]for an explanation.
} 
like to thank Marco Gualtieri for useful comments on an earlier version of this article.

A special thank goes to Lisa Jeffrey. The present work would not have been possible without her kind support.

\section{TWISTED EQUIVARIANT COHOMOLOGY}

2.1. Twisted cohomology, non-equivariant case. In this subsection, we briefly review the elements of twisted cohomology theory. We refer to AS05] for an excellent account of many fundamental aspects of the theory.

An H-twisted manifold $M$ is a smooth manifold in the usual sense together with a closed three form $\mathrm{H}$. The closed three form gives rise to a twisted differential operator $\mathrm{d}_{\mathrm{H}}$ defined by

$$
\mathrm{d}_{\mathrm{H}} \gamma=\mathrm{d} \gamma-\mathrm{H} \wedge \gamma, \quad \gamma \in \Omega(M) .
$$

It is easy to check directly that $d_{H}^{2}=0$. We define the H-twisted cohomology

$$
\mathrm{H}(\mathrm{M}, \mathrm{H})=\operatorname{kerd}_{\mathrm{H}} / \mathrm{imd}_{\mathrm{H}} \text {. }
$$

Given a two form $\lambda$ on $M$, then we can form the twisted differential $d_{H+d \lambda}$ and the group $\mathrm{H}(\mathrm{M}, \mathrm{H}+\mathrm{d} \lambda)$. There is an isomorphism

$$
\mathrm{H}(\mathrm{M}, \mathrm{H}) \rightarrow \mathrm{H}(\mathrm{M}, \mathrm{H}+\mathrm{d} \lambda), \quad[\alpha] \mapsto[\exp (\lambda) \alpha] .
$$

So any two closed differential three forms $H$ and $H^{\prime}$, if representing the same cohomology class, determine isomorphic groups $\mathrm{H}(\mathrm{M}, \mathrm{H})$ and $\mathrm{H}\left(\mathrm{H}, \mathrm{H}^{\prime}\right)$. However, as there are many two forms $\lambda$ such that $H^{\prime}=H+d \lambda$, these two groups are not uniquely isomorphic. Note also there is a homomorphism of groups

$$
H\left(M, H^{\prime}\right) \otimes H(M, H) \rightarrow H\left(M, H+H^{\prime}\right), \quad[\alpha] \otimes[\beta] \mapsto[\alpha \wedge \beta] .
$$

In particular, when $H^{\prime}=0$, this homomorphism gives $H(M, H)$ a natural $H(M)$-module structure: if $[\alpha] \in H(M)$ and $[\beta] \in H(M, H)$, then $[\alpha \wedge \beta] \in$ $\mathrm{H}(\mathrm{M}, \mathrm{H})$.

2.2. Equivariant de Rham theory. Let $\mathrm{G}$ be a compact connected Lie group and let $\Omega_{\mathrm{G}}(\mathrm{M})=\left(\mathrm{Sg}^{*} \otimes \Omega(\mathrm{M})\right)^{\mathrm{G}}$ be the Cartan complex of the G-manifold $M$. For brevity we will write $\Omega=\Omega(M)$ and $\Omega_{\mathrm{G}}=\Omega_{\mathrm{G}}(M)$ when there is no confusion. By definition an element of $\Omega_{\mathrm{G}}$ is an equivariant polynomial from $\mathfrak{g}$ to $\Omega$ and is called an equivariant differential form on $M$. The bigrading of the Cartan complex is defined by $\Omega_{G}^{i j}=\left(S^{i} \mathfrak{g}^{*} \otimes \Omega^{j-i}\right)^{G}$. It is equipped with a vertical differential $1 \otimes d$, which is usually abbreviated to $d$, and the horizontal differential $d^{\prime}$, which is defined by $d^{\prime} \alpha(\xi)=-l_{\xi_{M}} \alpha(\xi)$. Here $\iota_{\xi_{M}}$ denotes inner product with the vector field $\xi_{M}$ on $M$ induced by $\xi \in \mathfrak{g}$. As a total complex, $\Omega_{\mathrm{G}}$ has the grading

$$
\Omega_{G}^{k}=\bigoplus_{i+j=k} \Omega_{G}^{i j}
$$


and the total differential $d_{G}=d+d^{\prime}$. The total cohomology kerd $d_{G} / i d_{G}$ is the de Rham equivariant cohomology $\mathrm{H}_{\mathrm{G}}(M)$.

Now assume that the action of $G$ on $M$ is free. Choose a basis $\xi_{1}, \xi_{2}, \cdots, \xi_{k}$ of $\mathfrak{g}$. Then there exists $\theta^{1}, \theta^{2}, \cdots, \theta^{k} \in \Omega^{1}(M)$, called the connection elements, such that $\iota_{i, M} \theta^{j}=\delta_{j}^{i}$, where $\delta_{j}^{i}$ is the Kronecker symbol, and $\xi_{i, M}$ is the vector field on $M$ induced by $\xi_{i}$. Any differential form $\gamma \in \Omega(M)$ can be uniquely written as $\gamma=\gamma_{\text {hor }}+\sum_{\mathrm{I}} \theta^{\mathrm{I}} \gamma_{\mathrm{I}}$ such that $\iota_{\xi_{i, M}} \gamma_{\text {hor }}=0$ and $\iota_{\xi_{i, M}} \gamma_{\mathrm{I}}=0,1 \leq i \leq k$, where $\mathrm{I}$ is a multi-index, and $\gamma_{\text {hor }}$ is said to be the horizontal component of the form $\gamma$.

Let $c_{k, l}^{i}$ be the coefficient constants of the Lie algebra $\mathfrak{g}$, let

$$
c^{i}=d \theta^{i}+\frac{1}{2} c_{k, l}^{i} \theta^{k} \theta^{l}
$$

be the curvature elements, and let $\Omega(M)_{\text {bas }}$ be the space of basic forms in $\Omega(M)$, i.e., G-invariant forms which are annihilated by the vectors fields generated by the group action. The usual de Rham differential induces a differential $\mathrm{d}$ on $\Omega_{\text {bas }}$ and the cohomology of the differential complex $\left(\Omega_{\text {bas }}, d\right)$ is denoted by $\mathrm{H}\left(\Omega_{\text {bas }}\right)$. Then the Cartan map $\mathcal{C}: \Omega_{\mathrm{G}}(M) \rightarrow$ $\Omega(M)_{\text {bas, }}$ which is defined by

$$
\chi^{\mathrm{I}} \otimes \gamma \mapsto \mathrm{c}^{\mathrm{I}} \wedge \gamma_{\text {hor }}
$$

induces an isomorphism from $\mathrm{H}_{\mathrm{G}}(M)$ to $\mathrm{H}\left(\Omega_{\text {bas }}\right)$. More specifically, there is a chain homotopy operator $\mathrm{Q}: \Omega_{\mathrm{G}} \rightarrow \Omega_{\mathrm{G}}$ such that $\mathrm{d}_{\mathrm{G}} \mathrm{Q}-\mathrm{Q} \mathrm{d}_{\mathrm{G}}=\mathrm{id}-\mathcal{C}$. Define $\partial_{r}:=\frac{\partial}{\partial x^{r}}, 1 \leq r \leq k$, where $x^{1}, x^{2}, \cdots, x^{k}$ are inderterminates in the polynomial ring $\mathrm{Sg}^{*}$, and define the operators $\mathrm{K}$ and $\mathrm{R}$ on $\Omega_{\mathrm{G}}(\mathrm{M})$ by

$$
K:=-\theta^{r} \partial_{r}, \quad R:=d \theta^{r} \partial_{r} .
$$

Then the chain homotopy operator $\mathrm{Q}$ is given by

$$
\mathrm{Q}=\mathrm{KF}\left(\mathrm{I}+\mathrm{RF}+(\mathrm{RF})^{2}+\cdots\right)
$$

where $F$ is an operator on $\Omega_{G}(M)$ which preserves its bi-grading. We refer to [GS99, Chapter 5] for more details.

Let $\pi: M \rightarrow M / G$ be the quotient map. It is straightforward to check that the pull-back map $\pi^{*}: \mathrm{H}(M / \mathrm{G}) \rightarrow \mathrm{H}\left(\Omega_{\text {bas }}\right)$ is an isomorphism. In any event, we have the following result.

Theorem 2.1. ([GS99]) Assume the action of $\mathrm{G}$ on $\mathrm{M}$ is free. Then the Cartan map

$$
\Omega_{\mathrm{G}} \rightarrow \Omega(M)_{b a s}, \chi^{\mathrm{I}} \otimes \gamma \mapsto \mathrm{c}^{\mathrm{I}} \wedge \gamma_{\text {hor }},
$$

induces a natural isomorphism from $\mathrm{H}_{\mathrm{G}}(M)$ to the ordinary cohomology $\mathrm{H}(\mathrm{M} / \mathrm{G})$. 
2.3. Twisted equivariant cohomology. Throughout this subsection, we assume that $\mathrm{H}_{\mathrm{G}}$ is an equivariantly closed three form in the Cartan model $\Omega_{\mathrm{G}}(M)$. It gives rise to an $\mathrm{H}_{\mathrm{G}}$-twisted differential $\mathrm{d}_{\mathrm{G}, \mathrm{H}_{\mathrm{G}}}:=\mathrm{d}_{\mathrm{G}}-\mathrm{H}_{\mathrm{G}} \wedge$ on $\Omega_{\mathrm{G}}(M)$ such that $\mathrm{d}_{\mathrm{G}, \mathrm{H}_{\mathrm{G}}}^{2}=0$. We define

$$
\mathrm{H}\left(\Omega_{\mathrm{G}}(\mathrm{M}), \mathrm{H}_{\mathrm{G}}\right)=\operatorname{kerd}_{\mathrm{G}, \mathrm{H}_{\mathrm{G}}} / \operatorname{imd}_{\mathrm{G}, \mathrm{H}_{\mathrm{G}}} \text {. }
$$

Let $\widehat{\Omega}_{\mathrm{G}}=\left(\widehat{\mathrm{Sg}^{*}} \times \Omega(M)\right)^{\mathrm{G}}$. Here $\widehat{\mathrm{Sg}^{*}}=\mathbb{C}\left[\left[x_{1}, x_{2}, \cdots, x_{n}\right]\right]$ is the $a$ adic completion of the polynomial ring $S \mathfrak{g}^{*}$ with respect to the ideal $a=$ $\left(x_{1}, x_{2}, \cdots, x_{n}\right)$. It is easy to see that the $\mathrm{H}_{\mathrm{G}}$-twisted equivariant differential on $\Omega_{\mathrm{G}}$ extends naturally to a differential on $\widehat{\Omega}_{\mathrm{G}}$ which squares to zero. For brevity, this differential on $\widehat{\Omega}_{\mathrm{G}}$ is also denoted by $\mathrm{d}_{\mathrm{G}, \mathrm{H}_{\mathrm{G}}}$. The $\mathrm{H}_{\mathrm{G}}$-twisted equivariant cohomology is defined to be

$$
\mathrm{H}_{\mathrm{G}}\left(\mathrm{M}, \mathrm{H}_{\mathrm{G}}\right)=\operatorname{ker}\left(\widehat{\Omega}_{\mathrm{G}} \stackrel{\mathrm{d}_{\mathrm{G}, \mathrm{H}_{\mathrm{G}}}}{\longrightarrow} \widehat{\Omega}_{\mathrm{G}}\right) / \operatorname{im}\left(\widehat{\Omega}_{\mathrm{G}} \stackrel{\mathrm{d}_{\mathrm{G}, \mathrm{H}_{\mathrm{G}}}}{\longrightarrow} \widehat{\Omega}_{\mathrm{G}}\right) .
$$

In the special case $\mathrm{H}_{\mathrm{G}}=0$, the $\mathrm{H}_{\mathrm{G}}$-twisted equivariant cohomology is just $H\left(\widehat{\Omega}_{\mathrm{G}}(M), \mathrm{d}_{\mathrm{G}}\right)$, the equivariant cohomology with coefficients in the formal power series ring as we discussed in Appendix A.

Similar to the non-equivariant case, we have the following results.

Lemma 2.2. If $\lambda$ is an equivariant differential two form, then there is an isomorphism

$$
\mathrm{H}_{\mathrm{G}}\left(\mathrm{M}, \mathrm{H}_{\mathrm{G}}\right) \rightarrow \mathrm{H}_{\mathrm{G}}\left(\mathrm{M}, \mathrm{H}_{\mathrm{G}}+\mathrm{d}_{\mathrm{G}} \lambda\right), \quad[\alpha] \mapsto[\exp (\lambda) \alpha] .
$$

Thus two equivariantly closed three forms $\mathrm{H}_{\mathrm{G}}$ and $\mathrm{H}_{\mathrm{G}}^{\prime}$ representing the same equivariant cohomology class determine the isomorphic groups $\mathrm{H}_{\mathrm{G}}\left(\mathrm{M}, \mathrm{H}_{\mathrm{G}}\right)$ and $\mathrm{H}_{\mathrm{G}}\left(\mathrm{M}, \mathrm{H}_{\mathrm{G}}^{\prime}\right)$.

Lemma 2.3. Let $\mathrm{H}_{\mathrm{G}}$ and $\mathrm{H}_{\mathrm{G}}^{\prime}$ be two equivariantly closed three forms. If $\gamma$ is

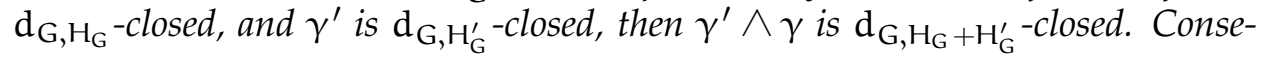
quently there is a homomorphism

$$
\mathrm{H}_{\mathrm{G}}\left(\mathrm{M}, \mathrm{H}_{\mathrm{G}}^{\prime}\right) \otimes \mathrm{H}_{\mathrm{G}}\left(\mathrm{M}, \mathrm{H}_{\mathrm{G}}\right) \rightarrow \mathrm{H}_{\mathrm{G}}\left(\mathrm{M}, \mathrm{H}_{\mathrm{G}}+\mathrm{H}_{\mathrm{G}}^{\prime}\right), \quad\left[\gamma^{\prime}\right] \otimes[\gamma] \mapsto\left[\gamma^{\prime} \wedge \gamma\right] .
$$

In particular, when $\mathrm{H}_{\mathrm{G}}^{\prime}=0$, the homomorphism

$$
\mathrm{H}\left(\widehat{\Omega}(M)_{\mathrm{G}}, \mathrm{d}_{\mathrm{G}}\right) \otimes \mathrm{H}_{\mathrm{G}}\left(\mathrm{M}, \mathrm{H}_{\mathrm{G}}\right) \rightarrow \mathrm{H}_{\mathrm{G}}\left(\mathrm{M}, \mathrm{H}_{\mathrm{G}}\right), \quad\left[\gamma^{\prime}\right] \otimes[\gamma] \mapsto\left[\gamma^{\prime} \wedge \gamma\right]
$$

defines an $\mathrm{H}\left(\Omega_{\mathrm{G}}(\mathrm{M})\right.$, $\left.\mathrm{d}_{\mathrm{G}}\right)$-module structure on $\mathrm{H}_{\mathrm{G}}\left(\mathrm{M}, \mathrm{H}_{\mathrm{G}}\right)$.

Note that since $\mathrm{H}\left(\widehat{\Omega}(M)_{G}, d_{G}\right)$ is a $\left(\widehat{S_{\mathfrak{g}^{*}}}\right)^{\mathrm{G}}$-module, c.f. Appendix $\mathrm{A}$, $\mathrm{H}_{\mathrm{G}}\left(\mathrm{M}, \mathrm{H}_{\mathrm{G}}\right)$ carries a $\left(\widehat{\mathrm{Sg}^{*}}\right)^{\mathrm{G}}$-module structure. Moreover, since $\left(\mathrm{Sg}^{*}\right)^{\mathrm{G}}$ is a subring of $\left(\widehat{\mathrm{Sg}^{*}}\right)^{\mathrm{G}}, \mathrm{H}_{\mathrm{G}}\left(\mathrm{M}, \mathrm{H}_{\mathrm{G}}\right)$ is also a $\left(\mathrm{Sg}^{*}\right)^{\mathrm{G}}$-module.

Now we describe the relationship between the twisted equivariant cohomology $\mathrm{H}_{\mathrm{G}}\left(\mathrm{M}, \mathrm{H}_{\mathrm{G}}\right)$ and $\mathrm{H}\left(\Omega_{\mathrm{G}}(\mathrm{M}), \mathrm{H}_{\mathrm{G}}\right)$. The sequence of modules

$$
S \mathfrak{g}^{*} \otimes \Omega(M) \supseteq \mathfrak{a}\left(\mathrm{Sg}^{*} \otimes \Omega(M)\right) \supseteq \cdots \supseteq \mathrm{a}^{\mathrm{k}}\left(\mathrm{Sg}^{*} \otimes \Omega(M)\right) \supseteq \cdots
$$


defines an a-adic topology on $\mathrm{Sg}^{*} \otimes \Omega(\mathrm{M})$, and so defines an a-adic topology on its subspaces $\Omega_{\mathrm{G}}=\left(\mathrm{Sg}^{*} \otimes \Omega(\mathrm{M})\right)^{\mathrm{G}}$ and $\operatorname{ker}\left(\Omega_{\mathrm{G}} \stackrel{\mathrm{d}_{\mathrm{G}, \mathrm{H}_{\mathrm{G}}}}{\longrightarrow} \Omega_{\mathrm{G}}\right)$. Then from the quotient map

$$
\operatorname{ker}\left(\Omega_{\mathrm{G}} \stackrel{\mathrm{d}_{\mathrm{G}, \mathrm{H}_{\mathrm{G}}}}{\longrightarrow} \Omega_{\mathrm{G}}\right) \rightarrow \mathrm{H}\left(\Omega_{\mathrm{G}}(\mathrm{M}), \mathrm{H}_{\mathrm{G}}\right)
$$

we get an a-adic topology on $\mathrm{H}\left(\Omega_{\mathrm{G}}(\mathrm{M}), \mathrm{H}_{\mathrm{G}}\right)$. It is straightforward to check by definition that the completion of $\mathrm{H}\left(\Omega(M), \mathrm{H}_{\mathrm{G}}\right)$ with respect to this topology also carries a $\left(\widehat{\mathrm{Sg}^{*}}\right)^{\mathrm{G}}$-module structure. The following result is a simple consequence of [AM99, Prop. 10.2].

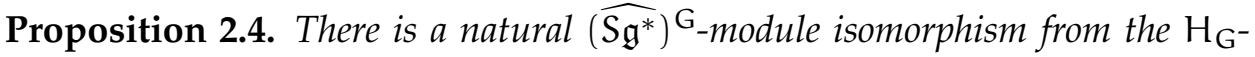
twisted equivariant cohomology $\mathrm{H}_{\mathrm{G}}\left(\mathrm{M}, \mathrm{H}_{\mathrm{G}}\right)$ to the completion of $\mathrm{H}\left(\Omega_{\mathrm{G}}(\mathrm{M}), \mathrm{H}_{\mathrm{G}}\right)$ with respect to the a-adic topology we described above.

We refer to [HuUribe06] for a detailed treatment of the foundational aspects of the twisted equivariant cohomology. For our purpose, we will need the following result later on.

Theorem 2.5. (Localization Theorem)([HuUribe06]) Suppose a compact connected torus $\mathrm{T}$ acts on a compact manifold $\mathrm{M}$. Then the kernel and cokernel of the canonical map

$$
i^{*}: \mathrm{H}_{\mathrm{T}}^{*}\left(M, \mathrm{H}_{\mathrm{T}}\right) \rightarrow \mathrm{H}_{\mathrm{T}}^{*}\left(\mathrm{M}^{\mathrm{T}}, i^{*} \mathrm{H}_{\mathrm{T}}\right)
$$

have support contained in $\bigcup_{H} \mathfrak{h}$, where $M^{\top}$ is the fixed points set of the torus $T$ action, $\mathrm{H}$ runs over the stabilizers $\neq \mathrm{T}$, and $\mathfrak{h}$ is the Lie algebra of $\mathrm{H}$.

Remark 2.6. Let $t^{*}$ be the dual space of the Lie algebra $t$ of $T$. Then $S t^{*}=$ $\mathbb{C}\left[x_{1}, x_{2}, \cdots, x_{n}\right]$ is a polynomial ring in $n$ variables. The support of an $S t^{*}$ module $A$ is defined to be $\operatorname{supp}(A)=\bigcap_{\left\{f \in S t^{*} \mid f \cdot A=0\right\}} V_{f}$, where $V_{f}=\{x \in$ $t \mid f(x)=0\}$. The support of $H_{T}^{*}\left(M, H_{T}\right)$ discussed in Theorem 2.5 is the support of it as an $\mathrm{St}^{*}$-module.

\section{GENERALIZED COMPLEX GEOMETRY}

Let $\mathrm{V}$ be an $\mathrm{n}$ dimensional vector space. There is a natural bi-linear pairing of type $(n, n)$ on $V \oplus V^{*}$ which is defined by

$$
\langle X+\alpha, Y+\beta\rangle=\frac{1}{2}(\beta(X)+\alpha(Y)) \text {. }
$$

A generalized complex structure on a vector space $V$ is an orthogonal linear map $\mathcal{J}: \mathrm{V} \oplus \mathrm{V}^{*} \rightarrow \mathrm{V} \oplus \mathrm{V}^{*}$ such that $\mathcal{J}^{2}=-1$. Let $\mathrm{L} \subset \mathrm{V}_{\mathbb{C}} \oplus \mathrm{V}_{\mathbb{C}}^{*}$ be the $\sqrt{-1}$ eigenspace of the generalized complex structure $\mathcal{J}$. Then $L$ is maximal isotropic and $\mathrm{L} \cap \overline{\mathrm{L}}=\{0\}$. Conversely, given a maximal isotropic $\mathrm{L} \subset \mathrm{V}_{\mathbb{C}} \oplus \mathrm{V}_{\mathbb{C}}^{*}$ so that $\mathrm{L} \cap \overline{\mathrm{L}}=\{0\}$, there exists an unique generalized complex structure $\mathcal{J}$ whose $\sqrt{-1}$ eigenspace is exactly L.

Let $\pi: V_{\mathbb{C}} \oplus \mathrm{V}_{\mathbb{C}}^{*} \rightarrow \mathrm{V}_{\mathbb{C}}$ be the natural projection. The type of $\mathcal{J}$ is the codimension of $\pi(L)$ in $V_{\mathbb{C}}$, where $L$ is the $\sqrt{-1}$ eigenspace of $\mathcal{J}$. 
Let $\sigma$ be the linear map on $\wedge \mathrm{V}^{*}$ which acts on decomposables by

$$
\sigma\left(v_{1} \wedge v_{2} \wedge \cdots \wedge v_{\mathrm{q}}\right)=v_{\mathrm{q}} \wedge v_{\mathrm{q}-1} \wedge \cdots \wedge v_{1},
$$

then we have the following bilinear pairing, called the Mukai pairing [Gua07], defined on $\wedge V^{*}$ :

$$
\left(\xi_{1}, \xi_{2}\right)=\left(\sigma\left(\xi_{1}\right) \wedge \xi_{2}\right)_{\text {top }},
$$

where $(\cdot, \cdot)_{\text {top }}$ indicates taking the top degree component of the form.

Let $B \in \wedge^{2} V^{*}$ be a two-form. For any $\xi \in \wedge V^{*}$, throughout this paper we will denote by $e^{\mathrm{B}} \xi$ the wedge product $e^{\mathrm{B}} \wedge \xi$. The following result was shown in [Gua07, Prop. 1.12].

\section{Proposition 3.1.}

$$
\left(e^{\mathrm{B}} \xi_{1}, e^{\mathrm{B}} \xi_{2}\right)=\left(\xi_{1}, \xi_{2}\right), \text { for any } \xi_{1}, \xi_{2} \in \wedge \mathrm{V}^{*},
$$

where $(\cdot, \cdot)$ denotes the Mukai pairing.

The Clifford algebra of $V_{\mathbb{C}} \oplus V_{\mathbb{C}}^{*}$ acts on the space of forms $\wedge V_{\mathbb{C}}^{*}$ via

$$
(X+\xi) \cdot \varphi=\iota_{X} \varphi+\xi \wedge \varphi .
$$

Since $\mathcal{J}$ is skew symmetric with respect to the natural pairing on $\mathrm{V} \oplus \mathrm{V}^{*}$, $\mathcal{J} \in \operatorname{so}\left(\mathrm{V} \oplus \mathrm{V}^{*}\right) \cong \Lambda^{2}\left(\mathrm{~V} \oplus \mathrm{V}^{*}\right) \subset \mathrm{CL}\left(\mathrm{V} \oplus \mathrm{V}^{*}\right)$. Therefore there is a Clifford action of $\mathcal{J}$ on the space of forms $\wedge \mathrm{V}^{*}$ ( and so on its complexification $\wedge \mathrm{V}_{\mathbb{C}}^{*}$ ) which determines an alternative grading : $\wedge V_{\mathbb{C}}^{*}=\bigoplus U^{k}$, where $U^{k}$ is the $-k \sqrt{-1}$ eigenspace of the Clifford action of $\mathcal{J}$.

Note also that a classical result of Chevalley [Che97] asserts that

$$
\left\{\varphi \in \wedge \mathrm{V}_{\mathbb{C}}^{*} \mid(X+\xi) \cdot \varphi=0, X+\xi \in \mathrm{L}\right\}
$$

is a one dimensional vector space in the space of exterior forms $\wedge V_{\mathbb{C}}^{*}$. Any form in this one dimensional subspace of $\wedge V_{\mathbb{C}}^{*}$ is said to be a pure spinor form associated to the generalized complex structure $\mathcal{J}$. On the other hand, given a form $\varphi \neq 0 \in \wedge \mathbb{V}_{\mathbb{C}}^{*} L_{\varphi}:=\left\{X+\xi \in V_{\mathbb{C}} \oplus V_{\mathbb{C}}^{*} \mid(X+\xi) \cdot \varphi=0\right\}$ is always an isotropic space. If in addition, $\mathrm{L}_{\varphi}$ is maximal isotropic, and if $(\varphi, \bar{\varphi}) \neq 0$, then $\mathrm{L}_{\varphi} \cap \overline{\mathrm{L}}_{\varphi}=\{0\}$ and there exists an unique generalized complex structure $\mathcal{J}$ whose $\sqrt{-1}$ eigenspace is exactly $\mathrm{L}_{\varphi}$.

Let $M$ be a manifold of dimension $n$. There is a natural pairing of type $(n, n)$ which is defined on $T M \oplus T^{*} M$ by

$$
\langle X+\alpha, Y+\beta\rangle=\frac{1}{2}(\beta(Y)+\alpha(X)),
$$

and which extends naturally to $\mathrm{T}_{\mathbb{C}} M \oplus \mathrm{T}_{\mathbb{C}}^{*} \mathrm{M}$.

For a closed three form $H$, the $H$-twisted Courant bracket of $T_{\mathbb{C}} M \oplus T_{\mathbb{C}}^{*} M$ is defined by the identity

$$
[X+\xi, Y+\eta]=[X, Y]+L_{X} \eta-L_{Y} \xi-\frac{1}{2} d(\eta(X)-\xi(Y))+\iota_{Y} \iota_{X} H .
$$

A generalized almost complex structure on a manifold $M$ is an orthogonal bundle map $\mathcal{J}: \mathrm{TM} \oplus \mathrm{T}^{*} \mathrm{M} \rightarrow \mathrm{TM} \oplus \mathrm{T}^{*} \mathrm{M}$ such that $\mathcal{J}^{2}=-1$. Moreover, 
$\mathcal{J}$ is an $\mathrm{H}$-twisted generalized complex structure if the sections of the $\sqrt{-1}$ eigenbundle of $\mathcal{J}$ is closed under the $\mathrm{H}$-twisted Courant bracket. The type of $\mathcal{J}$ at $m \in M$ is the type of the restricted generalized complex structure on $T_{m} M$.

The Clifford algebra of $C^{\infty}\left(T M \oplus T^{*} M\right)$ with the natural pairing acts on differential forms by

$$
(X+\xi) \cdot \varphi=\iota_{X} \varphi+\xi \wedge \varphi .
$$

If $\mathrm{L} \subset \mathrm{T}_{\mathbb{C}} M \oplus \mathrm{T}_{\mathbb{C}}^{*} \mathrm{M}$ is the $\sqrt{-1}$ eigenbundle of a generalized complex structure $\mathcal{J}$, then the differential forms annihilated by the Clifford action of the sections of $L$ span a line bundle sitting inside $\wedge T_{\mathbb{C}}^{*} M$, which is called the canonical line bundle of the generalized complex manifold $(M, \mathcal{J})$.

Since $\mathcal{J}$ can be identified with a smooth section of the Clifford bundle $\operatorname{CL}\left(\mathrm{TM} \oplus \mathrm{T}^{*} \mathrm{M}\right)$, there is a Clifford action of $\mathcal{J}$ on the space of differential forms. Let $U^{k}$ be the $-k \sqrt{-1}$ eigenbundle of $\mathcal{J}$. [Gua07] shows that there is a grading of the differential forms:

$$
\Omega^{*}(M)=\Gamma\left(\mathbf{U}^{-n}\right) \oplus \cdots \oplus \Gamma\left(\mathbf{U}^{0}\right) \oplus \cdots \oplus \Gamma\left(\mathbf{U}^{\mathrm{n}}\right) .
$$

It has been shown (See e.g. [Gua07] and [KL04]) that the integrability of an $\mathrm{H}$-twisted generalized complex structure $\mathcal{J}$ implies that

$$
\mathrm{d}_{\mathrm{H}}=\mathrm{d}-\mathrm{H} \wedge: \Gamma\left(\mathrm{U}^{\mathrm{k}}\right) \rightarrow \Gamma\left(\mathrm{U}^{\mathrm{k}-1}\right) \oplus \Gamma\left(\mathrm{U}^{\mathrm{k}+1}\right),
$$

which gives rise to operators $\partial$ and $\bar{\partial}$ via the projections

$$
\partial: \Gamma\left(\mathrm{U}^{\mathrm{k}}\right) \rightarrow \Gamma\left(\mathrm{U}^{\mathrm{k}-1}\right), \quad \bar{\partial}: \Gamma\left(\mathrm{U}^{\mathrm{k}}\right) \rightarrow \Gamma\left(\mathrm{U}^{\mathrm{k}+1}\right) .
$$

In this context, a twisted generalized complex manifold is said to satisfy the $\bar{\partial} \partial$-lemma if and only if

$$
\operatorname{ker} \partial \cap \operatorname{im} \bar{\partial}=\operatorname{im} \partial \cap \operatorname{ker} \bar{\partial}=\operatorname{im} \bar{\partial} \partial .
$$

Let $\Omega_{\bar{\partial}}(M)=\Omega(M) \cap$ ker $\bar{\partial}$. Since $d_{H}$ anti-commutes with $\bar{\partial},\left(\Omega_{\bar{\partial}}, d_{H}\right)$ is a differential complex with the differential $d_{H}$. The following result is a simple consequence of the $\bar{\partial} \partial$-lemma.

Proposition 3.2. (c.f., [Ca06, Thm. 4.1]) If the generalized complex manifold $(M, \mathcal{J})$ satisfies the $\bar{\partial}$-lemma, then the inclusion of the complex of $\bar{\partial}$-closed differential forms $\Omega_{\bar{\partial}}(M)$ into the complex of differential forms $\Omega(M)$ induces an isomorphism in cohomology:

$$
i:\left(\Omega_{\bar{\partial}}(M), d_{H}\right) \hookrightarrow\left(\Omega(M), d_{H}\right), i^{*}: H\left(\Omega_{\bar{\partial}}(M)\right) \cong H(M, H) .
$$

An H-twisted generalized Calabi-Yau structure $\mathcal{J}$ is a generalized complex structure such that its canonical line bundle admits a nowhere vanishing $d_{H}$-closed section $\rho$. On the other hand, given a nowhere vanishing $\mathrm{d}_{\mathrm{H}}$-closed differential form $\rho$ such that $(\rho, \bar{\rho}) \neq 0$, there exists a generalized Calabi-Yau structure $\mathcal{J}$ such that $\rho$ is the pure spinor associated to $\mathcal{J}$. By the abuse of the notation, we will also call such a differential form $\rho$ a generalized Calabi-Yau structure. 
Let $\mathrm{B}$ be a closed two-form on a manifold $\mathrm{M}$, and consider the orthogonal bundle map defined by

$$
e^{B}=\left(\begin{array}{ll}
1 & 0 \\
B & 1
\end{array}\right): \mathrm{TM} \oplus \mathrm{T}^{*} \mathrm{M} \rightarrow \mathrm{TM} \oplus \mathrm{T}^{*} \mathrm{M},
$$

where $B$ is regarded as a skew-symmetric map from TM to $T^{*} M$. This map preserves the canonical pairing $\langle\cdot, \cdot\rangle$ and the $\mathrm{H}$-twisted Courant bracket. As a consequence, if $\mathcal{J}$ is an $\mathrm{H}$-twisted generalized complex structure on $\mathrm{M}$, then $\mathcal{J}_{\mathrm{B}}:=e^{\mathrm{B}} \mathcal{J} e^{-\mathrm{B}}$ is another $\mathrm{H}$-twisted generalized complex structure on $\mathrm{M}$, called the B-transform of $\mathcal{J}$.

The effect of B-transforms on the canonical line bundle of a generalized complex structure has been studied in [Ca06].

Lemma 3.3. ([Ca06] ) Let B be a closed two form and let $\mathcal{J}_{\mathrm{B}}$ be the B-transform of the generalized Calabi-Yau structure $\mathcal{J}$ with a nowhere vanishing closed pure spinor $\rho$. Then $\mathcal{J}_{\mathrm{B}}$ is a generalized Calabi-Yau structure with a nowhere vanishing closed pure spinor $e^{-\mathrm{B}} \rho$.

Example 3.4. ([[H02], [Gua07])

- Let $(\mathrm{V}, \omega)$ be a $2 \mathrm{n}$ dimensional symplectic vector space. Then the map $\mathcal{J}: \mathrm{V} \oplus \mathrm{V}^{*} \rightarrow \mathrm{V} \oplus \mathrm{V}^{*}$ defined by

$$
\mathcal{J}=\left(\begin{array}{cc}
0 & -\omega^{-1} \\
\omega & 0
\end{array}\right)
$$

is a generalized complex structure on $\mathrm{V}$.

- Now let $(M, \omega)$ be a $2 n$ dimensional symplectic manifold. Then (3.3) defines a generalized complex structure $\mathcal{J}_{\omega}$ with the $\sqrt{-1}$ eigenbundle $L=\left\{X-\sqrt{-1} \iota_{X} \omega \mid X \in T_{\mathbb{C}} M\right\}$. It is easy to check that the canonical line bundle admits a nowhere vanishing closed section $e^{i \omega}$. And so $\mathcal{J}_{\omega}$ is a generalized Calabi-Yau structure. On the other hand, the following lemma shows that any nowhere vanishing closed section of the canonical line bundle of $\mathcal{J}_{\omega}$ must be of the form $a e^{i \omega}$ for some non-zero constant $a$.

Lemma 3.5. Let $(\mathrm{M}, \omega)$ be a $2 \mathrm{n}$ dimensional connected symplectic manifold and $\mathcal{J}_{\omega}$ the generalized complex structure defined as in (3.3). Suppose $\rho$ is a nowhere vanishing closed pure spinor associated to the generalized Calabi-Yau structure $\mathcal{J}_{\omega}$. Then we have $\rho=a e^{i \omega}$ for some non-zero constant $a$.

Proof. By assumption we have $\rho=f e^{i \omega}$ for some nowhere vanishing function $f \in C^{\infty}(M)$. Since $d \rho=0$, we get $d f \wedge e^{i \omega}=0$. In particular, we have

$$
(i \omega)^{n-1} \wedge d f=0 .
$$

It is well known that the Lefschetz map

$$
\mathrm{L}_{\omega}^{n-1}: \Omega^{1}(M) \rightarrow \Omega^{2 n-1}(M), \eta \mapsto \omega^{n-1} \wedge \eta
$$


is an isomorphism, c.f., [Yan96, Corollary 2.7]. It follows that $\mathrm{df}=0$ and so $f=a$ for some non-zero constant $a$. This finishes the proof.

It is important for our purpose to notice that given a $2 \mathrm{n}$ dimensional $\mathrm{H}$-twisted generalized Calabi-Yau manifold $M$, the generalized Calabi-Yau structure $\rho$ on $M$ gives rise to a volume form

$$
\frac{(-1)^{n}}{(2 i)^{n}}(\rho, \bar{\rho})
$$

where $(\cdot, \cdot)$ denotes the Mukai pairing. Note that if $M$ is a symplectic manifold with a symplectic form $\omega$ and the generalized Calabi-Yau structure is given by $e^{i \omega}$, then up to a normalization factor the volume form we defined above coincides with the symplectic volume $\frac{1}{n !} \omega^{n}$.

A manifold $M$ is said to be an H-twisted generalized Kähler manifold if it has two commuting $\mathrm{H}$-twisted generalized complex structures $\mathcal{J}_{1}, \mathcal{J}_{2}$ such that $\left\langle-\mathcal{J}_{1} \mathcal{J}_{2} \xi, \xi\right\rangle>0$ for any $\xi \neq 0 \in \mathrm{C}^{\infty}\left(\mathrm{T}_{\mathbb{C}} M \oplus \mathrm{T}_{\mathbb{C}}^{*} \mathrm{M}\right)$, where $\langle\cdot, \cdot\rangle$ is the canonical pairing on $T_{\mathbb{C}} M \oplus T_{\mathbb{C}}^{*} M$. The following remarkable result is due to Gualtieri.

Theorem 3.6. ([Gua04]) Assume that $\left(M, \mathcal{J}_{1}, \mathcal{J}_{2}\right)$ is a compact H-twisted generalized Kähler manifold. Then it satisfies the $\bar{\partial}$-lemma with respect to both $\mathcal{J}_{1}$ and $\mathcal{J}_{2}$.

\section{THE TWISTED EQUIVARIANT COHOMOLOGY OF HAMILTONIAN ACTIONS ON TWISTED GENERALIZED COMPLEX MANIFOLDS}

First we recall the definition of Hamiltonian actions on $\mathrm{H}$-twisted generalized complex manifolds given in [LT05].

Definition 4.1. 4 [LT05]) Let a compact Lie group $\mathrm{G}$ with Lie algebra $\mathfrak{g}$ act on a manifold $\mathrm{M}$, preserving an $\mathrm{H}$-twisted generalized complex structure $\mathcal{J}$, where $\mathrm{H} \in \Omega^{3}(\mathrm{M})^{\mathrm{G}}$ is closed. Let $\mathrm{L} \subset \mathrm{T}_{\mathbb{C}} \mathrm{M} \oplus \mathrm{T}_{\mathbb{C}}^{*} \mathrm{M}$ denote the $\sqrt{-1}$ eigenbundle of $\mathcal{J}$. The action of $\mathrm{G}$ is said to be Hamiltonian if there exists a smooth equivariant function $\mu: M \rightarrow \mathfrak{g}^{*}$, called the generalized moment map, and a one form $\alpha \in \Omega^{1}\left(M, \mathfrak{g}^{*}\right)$, called the moment one form, so that

a) $\xi_{M}-\sqrt{-1}\left(\mathrm{~d} \mu^{\xi}+\sqrt{-1} \alpha^{\xi}\right)$ lies in $\mathrm{C}^{\infty}(\mathrm{L})$ for all $\xi \in \mathfrak{g}$, where $\xi_{M}$ denotes the induced vector field.

b) $\mathrm{H}+\alpha$ is an equivariantly closed three form in the usual Cartan Model.

Therefore given a Hamiltonian action on an $\mathrm{H}$-twisted generalized complex manifold, there is an equivariantly closed three form $\mathrm{H}_{\mathrm{G}}:=\mathrm{H}+\alpha$ which is part of the data defining the Hamiltonian action.

Next we recall the definition of the generalized equivariant cohomology as introduced in [Lin06] and compare it with the twisted equivariant cohomology $\mathrm{H}_{\mathrm{G}}\left(\mathrm{M}, \mathrm{H}_{\mathrm{G}}\right)$. First observe that since $\mathrm{H}$ is an invariant three form,

\footnotetext{
${ }^{4}$ Indeed, Condition (b) was not imposed in [LT05, Definition A.2.]. However, in order to make the quotient construction work, Tolman and the author made it clear in [LT05, Prop. A.7, A.10] that $\mathrm{H}+\alpha$ must be equivariantly closed in the usual Cartan model.
} 
the operators $d_{H}=d-H \wedge, \bar{\partial}$, and $\partial$ are G-equivariant and thus have natural extensions to the space of the usual equivariant differential forms, i.e., the Cartan model $\Omega_{\mathrm{G}}$. For brevity, we will also denote these extensions by $d_{H}, \bar{\partial}$ and $\partial$. To encode the information given by the moment one form, [Lin06] introduced two equivariant differentials $\mathrm{D}_{\mathrm{G}}=\mathrm{d}_{\mathrm{H}}+\mathcal{A}$ and $\bar{\partial}_{\mathrm{G}}=\bar{\partial}+\mathcal{A}$ in the Cartan model, where $\mathcal{A}$ is defined by

$$
(\mathcal{A} \gamma)(\xi)=-\mathfrak{l}_{\xi_{M}} \gamma(\xi)+\sqrt{-1}\left(\mathrm{~d} \mu^{\xi}+\sqrt{-1} \alpha^{\xi}\right) \wedge \gamma(\xi), \quad \xi \in \mathfrak{g}, \quad \gamma \in \Omega_{\mathrm{G}} .
$$

It is easy to check that $D_{G}^{2}=0$ and $\bar{\partial}_{G}^{2}=0$. In particular, we have the following definition for the generalized equivariant cohomology.

Definition 4.2. Let $\Omega_{\mathrm{G}}=\left(\mathrm{Sg}^{*} \otimes \Omega(\mathrm{M})\right)^{\mathrm{G}}$ be $\mathrm{Z}_{2}$ graded. Then $\mathrm{D}_{\mathrm{G}}=\mathrm{d}_{\mathrm{H}}+\mathcal{A}$ is a differential of degree 1 . And the $Z_{2}$ graded generalized equivariant cohomology is defined to be

$$
\mathrm{H}^{\text {even } / \text { odd }}\left(\Omega_{\mathrm{G}}, \mathrm{D}_{\mathrm{G}}\right)=\frac{\operatorname{ker}\left(\Omega_{\mathrm{G}}^{\text {even/odd }} \stackrel{\mathrm{D}_{\mathrm{G}}}{\longrightarrow} \Omega_{\mathrm{G}}^{\text {odd/even }}\right)}{i m\left(\Omega_{\mathrm{G}}^{\text {odd/even }} \stackrel{\mathrm{D}_{\mathrm{G}}}{\longrightarrow} \Omega_{\mathrm{G}}^{\text {even/odd }}\right)} .
$$

Note that the differential operator $\mathrm{D}_{\mathrm{G}}$ on $\Omega_{\mathrm{G}}$ extends naturally to a differential operator on $\widehat{\Omega}_{\mathrm{G}}$, which we will also denote by $\mathrm{D}_{\mathrm{G}}$. Define

$$
H\left(\widehat{\Omega}_{G}, D_{G}\right)=\frac{\operatorname{ker}\left(\widehat{\Omega}_{G} \stackrel{D_{G}}{\longrightarrow} \widehat{\Omega}_{G}\right)}{\operatorname{im}\left(\widehat{\Omega}_{G} \stackrel{D_{G}}{\longrightarrow} \widehat{\Omega}_{G}\right)} .
$$

By the discussion in Section 2.3, it is clear that the differential operator $\mathrm{D}_{\mathrm{G}}$ on $\widehat{\Omega}_{\mathrm{G}}$ is nothing but the the usual equivariant differential $\mathrm{d}_{\mathrm{G}}$ twisted by the equivariantly closed three form $\mathrm{H}_{\mathrm{G}}-\sqrt{-1} \mathrm{~d}_{\mathrm{G}} \mu$. (Note that $\mathrm{d} \mu=\mathrm{d}_{\mathrm{G}} \mu$ is an exact equivariant three form.) The following result is a simple consequence of Lemma2.2.

Proposition 4.3. Consider the Hamiltonian action of a compact connected Lie group $\mathrm{G}$ on an $\mathrm{H}$-twisted generalized complex manifold with generalized moment map $\mu: M \rightarrow \mathfrak{g}^{*}$. Then the map

$$
\mathrm{H}_{\mathrm{G}}\left(\mathrm{M}, \mathrm{H}_{\mathrm{G}}\right) \rightarrow \mathrm{H}\left(\widehat{\Omega}_{\mathrm{G}}, \mathrm{D}_{\mathrm{G}}\right), \quad[\gamma] \mapsto[\exp (-\sqrt{-1} \mu) \gamma]
$$

defines an isomorphism between the $\mathrm{H}_{\mathrm{G}}$-twisted equivariant cohomology $\mathrm{H}_{\mathrm{G}}\left(\mathrm{M}, \mathrm{H}_{\mathrm{G}}\right)$ and the generalized equivariant cohomology.

Recall that the following result is proved in [Lin06], which is a direct generalization of an immediate consequence of the $d_{G} \delta$-lemma [LS03] in symplectic geometry.

Theorem 4.4. If we assume that the $\mathrm{H}$-twisted generalized complex manifold $\mathrm{M}$ satisfies the $\bar{\partial} \partial$-lemma, and assume the action of a compact connected Lie group $\mathrm{G}$ on $\mathrm{M}$ is Hamiltonian, then as $\left(\mathrm{Sg}^{*}\right)^{\mathrm{G}}$-modules, $\mathrm{H}\left(\Omega_{\mathrm{G}}, \mathrm{D}_{\mathrm{G}}\right) \cong\left(\mathrm{Sg}^{*}\right)^{\mathrm{G}} \otimes$ $\mathrm{H}(\mathrm{M}, \mathrm{H})$. 
Using an argument similar to the one we give to prove Proposition 2.4 , it is easy to see that there is a natural $\left(\widehat{\mathrm{Sg}^{*}}\right) \mathrm{G}_{\text {-module isomorphism from }}$ $\mathrm{H}\left(\widehat{\Omega}_{\mathrm{G}}, \mathrm{D}_{\mathrm{G}}\right)$ to $\left(\widehat{\mathrm{Sg}^{*}}\right)^{\mathrm{G}} \otimes \mathrm{H}(\mathrm{M}, \mathrm{H})$ by Theorem 4.4. Combining this fact with Proposition 4.3 , we have the following result.

Theorem 4.5. (Equivariant Formality) Assume the $\mathrm{H}$-twisted generalized complex manifold $M$ satisfies the $\bar{\partial} \partial$-lemma, and assume the action of a compact connected Lie group $\mathrm{G}$ on $\mathrm{M}$ is Hamiltonian. Let $\alpha$ be the moment one form of the Hamiltonian action and let $\mathrm{H}_{\mathrm{G}}=\mathrm{H}+\alpha$. Then as $\left(\widehat{\mathrm{Sg}^{*}}\right)^{\mathrm{G}}$-modules we have that

$$
\mathrm{H}_{\mathrm{G}}\left(\mathrm{M}, \mathrm{H}_{\mathrm{G}}\right) \cong\left(\widehat{\mathrm{Sg}^{*}}\right)^{\mathrm{G}} \otimes \mathrm{H}(\mathrm{M}, \mathrm{H}),
$$

where the isomorphism depends only on the generalized moment map.

It is interesting to give a more concrete description of the isomorphism (4.2). Note that via the isomorphism (4.2) one gets a canonical map

$$
\mathrm{s}: \mathrm{H}(\mathrm{M}, \mathrm{H}) \rightarrow \mathrm{H}_{\mathrm{G}}\left(\mathrm{M}, \mathrm{H}_{\mathrm{G}}\right)
$$

such that the inverse of the canonical isomorphism (4.2) is given by $1 \otimes \mathrm{s}$. Let us show how in principle one can compute the map s.

Let $\varphi$ be a $\mathrm{d}_{\mathrm{H}^{-}}$closed differential form representing a cohomology class in $H(M, H)$. Since the generalized complex manifold $M$ satisfies the $\bar{\partial} \partial-$ lemma, by Proposition 3.2 we may assume that $\varphi$ is both $\bar{\partial}$ and $\partial$ closed. We claim that we may assume that $\varphi$ is G-invariant as well. Resorting to the usual averaging trick, to establish the claim one needs only to check that the induced action of $\mathrm{G}$ on $\mathrm{H}(\mathrm{M}, \mathrm{H})$ is trivial. Since $\mathrm{G}$ is connected, it suffices to show that all operators $L_{\xi_{M}}$ act trivially on $H(M, H)$, where $\xi_{M}$ denotes the vector field on $M$ induced by $\xi \in \mathfrak{g}$. However, since $\iota_{\xi_{M}} \mathrm{H}=\mathrm{d} \alpha^{\xi}$, we have

$$
\begin{aligned}
\mathrm{L}_{\xi_{M}} & =d \iota_{\xi_{M}}+\iota_{\xi_{M}} \mathrm{~d}=(\mathrm{d}-\mathrm{H} \wedge)\left(\iota_{\xi_{M}}+\alpha^{\xi} \wedge\right)+\left(\iota_{\xi_{M}}+\alpha^{\xi} \wedge\right)(\mathrm{d}-\mathrm{H} \wedge) \\
& =\mathrm{d}_{\mathrm{H}}\left(\iota_{\xi_{M}}+\alpha^{\xi} \wedge\right)+\left(\iota_{\xi_{M}}+\alpha^{\xi} \wedge\right) d_{H}
\end{aligned}
$$

That is to say that $L_{\xi_{M}}$ is chain homotopic to zero in $H(M, H)$ and our claim is established. In view of the grading (3.2), write $\varphi=\sum_{i} \varphi^{i}$, where $\varphi^{i} \in$ $\Gamma\left(U^{i}\right)$. Then each homogenous component $\varphi^{i}$ is G-invariant and is both $\bar{\partial}$ and $\partial$ closed.

Observe the grading (3.2) on the space of differential forms induces an alternative filtration on the Cartan model

$$
\mathrm{F}^{\mathrm{k}} \Omega_{\mathrm{G}}=\bigoplus_{j \leq k}\left(\mathrm{Sg}^{*} \otimes \Gamma\left(\mathrm{u}^{\mathrm{j}}\right)\right)^{\mathrm{G}} \text {. }
$$

Now fix an $i$ for the moment. It follows from [Lin06, Lemma 4.9] that $\mathcal{A} \varphi^{i}$ is $\partial$-exact. Since $\bar{\partial}$ anti-commutes with $\mathcal{A}, \mathcal{A} \varphi^{i} \in \mathrm{F}^{i-1} \Omega_{\mathrm{G}}$ is both $\partial$ exact and $\bar{\partial}$-closed. It then follows from the $\bar{\partial} \partial$-lemma that there exists $\gamma^{i-1} \in \mathrm{F}^{i-1} \Omega_{\mathrm{G}}$ such that $\mathcal{A} \varphi^{i}=\bar{\partial} \partial \gamma^{i-1}$. ( We refer to [Lin06, Lemma 4.9] for a detailed proof of this fact.) Also $\mathcal{A} \partial \gamma^{i-1}=-\partial \mathcal{A} \gamma^{i-1}$ is both $\partial$ exact and $\bar{\partial}$-closed. Another application of the $\bar{\partial} \partial$-lemma implies that there exists 
$\gamma^{i-3} \in \mathrm{F}^{\mathrm{i}-3} \Omega_{\mathrm{G}}$ such that $\mathcal{A} \partial \gamma^{\mathrm{i}-1}=\bar{\partial} \partial \gamma^{i-3}$. Since the filtration (4.3) is finite, applying this argument recursively we get a finite chain $\gamma^{k} \in \mathrm{F}^{i+1-2 k} \Omega_{\mathrm{G}}$ such that

$$
\varphi_{g}^{i}:=\varphi^{i}+\sum_{k} \partial \gamma^{k}
$$

is $\bar{\partial}_{G}=(\bar{\partial}+\mathcal{A})$-closed. Note that by construction $\varphi_{g}^{i}$ is also $\partial$-closed. So $\varphi_{g}^{i}$ is $D_{G}=\left(\bar{\partial}_{G}+\partial\right)$-closed. Thus by Proposition $4.3 e^{\sqrt{-1} \mu}\left(\varphi_{\mathrm{g}}^{i}\right)$ is $d_{G, H_{G}}$-closed in $\widehat{\Omega}_{\mathrm{G}}$, the completion of $\Omega_{\mathrm{G}}$. Set $\varphi_{\mathrm{g}}:=\sum_{i} \varphi_{\mathrm{g}}^{\mathrm{i}}$. Then $e^{\sqrt{-1} \mu} \varphi_{\mathrm{g}}$ is a $\mathrm{d}_{\mathrm{G}, \mathrm{H}_{\mathrm{G}}}{ }^{-}$ closed element in $\widehat{\Omega}_{\mathrm{G}}$, representing a cohomology class in $\mathrm{H}_{\mathrm{G}}\left(\mathrm{M}, \mathrm{H}_{\mathrm{G}}\right)$. A careful reading of the proof of Theorem 4.4 given in [Lin06] shows that $s[\varphi]=\left[\varphi_{\mathrm{g}}\right]$.

Recall that an equivariant differential form $\eta \in \Omega_{\mathrm{G}}(M)$ can be regarded as a polynomial mapping

$$
\mathfrak{g} \rightarrow \Omega(M)
$$

Thus there is a natural map

$$
p: \Omega_{\mathrm{G}}(M) \rightarrow \Omega(M), \eta \mapsto \eta(0),
$$

where $\eta(0)$ is the value of the polynomial map $\eta: \mathfrak{g} \rightarrow \Omega(M)$ evaluated at the zero element in $\mathfrak{g}$. It is easy to see that $p$ is a chain map between the differential complexes $\left(\widehat{\Omega}_{\mathrm{G}}(\mathrm{M}), \mathrm{d}_{\mathrm{G}, \mathrm{H}_{\mathrm{G}}}\right)$ and $\left(\Omega(\mathrm{M}), \mathrm{d}_{\mathrm{H}}\right)$ and so induces a map

$$
\widetilde{\mathrm{p}}: \mathrm{H}_{\mathrm{G}}\left(\mathrm{M}, \mathrm{H}_{\mathrm{G}}\right) \rightarrow \mathrm{H}(\mathrm{M}, \mathrm{H})
$$

furthermore, by the above description of the map s, one checks easily that $\widetilde{p} s=\mathrm{id}$. In particular, we have proved the following result.

Corollary 4.6. The canonical map s is a section of $\widetilde{p}$. Thus every cohomology class in $\mathrm{H}(\mathrm{M}, \mathrm{H})$ has a canonical equivariant extension in $\mathrm{H}_{\mathrm{G}}\left(\mathrm{M}, \mathrm{H}_{\mathrm{G}}\right)$.

Recall from [LT05] that the action of a compact connected Lie group G on an H-twisted generalized Kähler manifold $\left(M, \mathcal{J}_{1}, \mathcal{J}_{2}\right)$ is said to be Hamiltonian if the action preserves the generalized Kähler pair $\left(\mathcal{J}_{1}, \mathcal{J}_{2}\right)$ and if there exist a generalized moment map and a moment one-form for the twisted generalized complex structure $\mathcal{J}_{1}$. By Theorem 3.6 an $\mathrm{H}$-twisted generalized Kähler manifold always satisfies the $\bar{\partial} \partial$-lemma with respect to both $\mathcal{J}_{1}$ and $\mathcal{J}_{2}$. The following result is a direct consequence of Theorem 4.5.

Theorem 4.7. Suppose that a compact connected Lie group $\mathrm{G}$ acts on an $\mathrm{H}-$ twisted generalized Kähler manifold $\left(M, \mathcal{J}_{1}, \mathcal{J}_{2}\right)$ in a Hamiltonian fashion. Let $\alpha$ be the moment one form and $\mathrm{H}_{\mathrm{G}}=\mathrm{H}+\alpha$ the equivariantly closed three form. Then we have

$$
\mathrm{H}_{\mathrm{G}}\left(\mathrm{M}, \mathrm{H}_{\mathrm{G}}\right) \cong\left(\widehat{\mathrm{Sg}^{*}}\right)^{\mathrm{G}} \otimes \mathrm{H}(\mathrm{M}, \mathrm{H}),
$$

where the isomorphism is an isomorphism of $\left(\widehat{\mathrm{Sg}^{*}}\right)^{\mathrm{G}}$-modules and depends only on the generalized moment map. 
Next we extend the Kirwan injectivity theorem [Kir86] to the case of Hamiltonian actions on H-twisted generalized Kähler manifolds. We need the following intermediate result.

Lemma 4.8. Suppose that a compact connected torus T acts on a generalized complex manifold $(\mathrm{M}, \mathcal{J})$ in a Hamiltonian fashion. And suppose that $W$ is a connected component of the fixed points set $\mathrm{M}^{\top}$. Then $\mathrm{W}$ is a generalized complex manifold itself and the induced trivial T-action on $\mathrm{W}$ is Hamiltonian.

Proof. Let $L$ be the $\sqrt{-1}$ eigenbundle of $\mathcal{J}: \mathrm{T}_{\mathbb{C}} M \oplus \mathrm{T}_{\mathbb{C}}^{*} \mathrm{M} \rightarrow \mathrm{T}_{\mathbb{C}} \mathrm{M} \oplus \mathrm{T}_{\mathbb{C}}^{*} \mathrm{M}$. It is proved in [Lin06, Lemma 5.4] that $X$ carries a generalized complex structure whose $\sqrt{-1}$ eigenbudle is

$$
\mathrm{L}_{W, x}=\left\{\mathrm{X}+\left(\left.\xi\right|_{\mathbb{C}_{\mathbb{W}}}\right) \mid \mathrm{X}+\xi \in \mathrm{L} \cap\left(\mathrm{T}_{\mathbb{C}, x} \mathrm{~W} \oplus \mathrm{T}_{\mathbb{C}, x}^{*} \mathrm{M}\right)\right\} .
$$

Let $\mu: M \rightarrow \mathfrak{t}^{*}$ and $\alpha \in \Omega^{1}\left(M, \mathfrak{t}^{*}\right)$ be the generalized moment map and the moment one form of the T action on $M$ respectively. Let $\mu_{W}$ and $\alpha_{W}$ be the restrictions of $\mu$ and $\alpha$ to $W$. Then it is easy to check that $\sqrt{-1}\left(d \mu_{W}^{\xi}+\right.$ $\left.\sqrt{-1} \alpha_{W}^{\xi}\right) \in \mathrm{C}^{\infty}\left(\mathrm{L}_{W}\right)$ for any $\xi \in \mathfrak{t}$. This proves that the trivial $\mathrm{T}$ action on $W$ is Hamiltonian.

Theorem 4.9. (Kirwan injectivity) Suppose that a compact connected Lie group $\mathrm{G}$ acts on an $\mathrm{H}$-twisted generalized Kähler manifold $\left(M, \mathcal{J}_{1}, \mathcal{J}_{2}\right)$ in a Hamiltonian fashion. Then the canonical map

$$
i^{*}: \mathrm{H}_{\mathrm{T}}^{*}\left(M, \mathrm{H}_{\mathrm{T}}\right) \rightarrow \mathrm{H}_{\mathrm{T}}^{*}\left(M^{\top}, i^{*} \mathrm{H}_{\mathrm{T}}\right)
$$

induced by the inclusion $i: M^{\top} \rightarrow M$ is injective, where $M^{\top}$ is the fixed points set of the torus $\mathrm{T}$ action.

Proof. It is proved in [Lin06, Prop. 5.5] any connected component of the fixed points set is a generalized Kähler manifold itself. Combining this fact with Lemma4.8 and Theorem 4.7, one sees immediately that $\mathrm{H}_{\mathrm{T}}\left(M^{\top}, i^{*} \mathrm{H}_{\mathrm{T}}\right)$ is a free $\widehat{S \mathfrak{t}^{*}}$-module. Since $S \mathfrak{t}^{*}$ is a subring of $\widehat{S \mathfrak{t}^{*}}, \mathrm{H}_{\mathrm{T}}\left(\mathrm{M}^{\top}, i^{*} \mathrm{H}_{\mathrm{T}}\right)$ must be a free $\mathrm{St}^{*}$-module as well. So if $[\alpha]$ is a torsion element in $\mathrm{H}_{T}\left(\mathrm{M}, \mathrm{H}_{\mathrm{T}}\right)$, it gets mapped to zero in $\mathrm{H}_{\mathrm{T}}^{*}\left(M^{\mathrm{T}}, i^{*} \mathrm{H}_{\mathrm{T}}\right)$. On the other hand, Theorem 2.5 implies that the kernel of the map $i^{*}$ is a torsion module. So the kernel of $i^{*}$ is the module of torsion elements in $\mathrm{H}_{\mathrm{T}}\left(\mathrm{M}, \mathrm{H}_{\mathrm{T}}\right)$. However, by Theorem 4.7 $\mathrm{H}_{\mathrm{T}}\left(\mathrm{M}, \mathrm{H}_{\mathrm{T}}\right)$ is a free $\widehat{\mathrm{St}^{*}}$-module. So it must be a free $\mathrm{St}^{*}$-module and it does not have any non-trivial torsion elements. We conclude that the map $i^{*}$ is injective.

\section{SOME REFLECTIONS ON THE COHOMOLOGY OF GENERALIZED COMPLEX QUOTIENTS}

Let a compact Lie group $\mathrm{G}$ act on a twisted generalized complex manifold $(M, \mathcal{J})$ with generalized moment map $\mu$. Let $\mathcal{O}_{a}$ be the co-adjoint orbit through $a \in \mathfrak{g}^{*}$. If $\mathrm{G}$ acts freely on $\mu^{-1}\left(\mathcal{O}_{a}\right)$, then $\mathcal{O}_{a}$ consists of regular values and $M_{a}=\mu^{-1}\left(\mathcal{O}_{a}\right) / G$ is a manifold, which is called the generalized complex quotient. The following two results were proved in [LT05]. 
Lemma 5.1. Let a compact Lie group $\mathrm{G}$ act freely on a manifold $\mathrm{M}$. Let $\mathrm{H}$ be an invariant closed three form and let $\alpha$ be an equivariant mapping from $\mathfrak{g}$ to $\Omega^{1}(M)$. Fix a connection $\theta \in \Omega\left(M, \mathfrak{g}^{*}\right)$. Then if $\mathrm{H}+\alpha \in \Omega_{\mathrm{G}}^{3}(\mathrm{M})$ is equivariantly closed, there exists a natural form $\Gamma \in \Omega^{2}(M)^{\mathrm{G}}$ so that $\iota_{\mathrm{M}} \Gamma=\alpha^{\xi}$. Thus $\mathrm{H}+\alpha+$ $\mathrm{d}_{\mathrm{G}} \Gamma \in \Omega^{3}(\mathrm{M})^{\mathrm{G}} \subset \Omega_{\mathrm{G}}^{3}(\mathrm{M})$ is closed and basic and so descends to a closed form $\widetilde{\mathrm{H}} \in \Omega^{3}(\mathrm{M} / \mathrm{G})$ so that $[\widetilde{\mathrm{H}}]$ is the image of $[\mathrm{H}+\alpha]$ under the Kirwan map.

Proposition 5.2. Assume there is a Hamiltonian action of a compact Lie group $\mathrm{G}$ on an $\mathrm{H}$-twisted generalized complex manifold $(\mathrm{M}, \mathcal{J})$ with generalized moment map $\mu: M \rightarrow \mathfrak{g}^{*}$ and moment one-form $\alpha \in \Omega^{1}\left(\mathrm{M}, \mathfrak{g}^{*}\right)$. Let $\mathcal{O}_{\mathrm{a}}$ be a co-adjoint orbit through $\mathrm{a} \in \mathfrak{g}^{*}$ so that $\mathrm{G}$ acts freely on $\mu^{-1}\left(\mathcal{O}_{\mathrm{a}}\right)$. Given a connection on $\mu^{-1}\left(\mathcal{O}_{a}\right)$, the generalized complex quotient $M_{a}$ inherits an $\widetilde{H}$-twisted generalized complex structure $\widetilde{\mathcal{J}}$, where $\widetilde{\mathrm{H}}$ is defined as in the Lemma 5.1 Up to B-transform, $\widetilde{\mathrm{J}}$ is independent of the choice of connection. Finally, for all $\mathrm{m} \in \mu^{-1}\left(\mathcal{O}_{\mathrm{a}}\right)$,

$$
\operatorname{type}(\tilde{\mathcal{J}})_{[\mathrm{m}]}=\operatorname{type}(\mathcal{J})_{\mathrm{m}} \text {. }
$$

It is an interesting question if we can read off the cohomology of the generalized complex quotient $M_{a}$ from the equivariant cohomology of the Hamiltonian generalized complex manifold $M$. The Kirwan map is the key to understanding this question.

Assume that $\mathrm{G}$ acts freely on $\mu^{-1}\left(\mathcal{O}_{a}\right)$. Let $i: \mu^{-1}\left(\mathcal{O}_{a}\right) \rightarrow M$ be the inclusion map and $\pi: \mu^{-1}\left(\mathcal{O}_{a}\right) \rightarrow M_{a}$ the quotient map. Then there is a Kirwan map $k: \mathrm{H}_{\mathrm{G}}(M) \rightarrow \mathrm{H}\left(M_{\mathrm{a}}\right)$ such that the following diagram commutes:

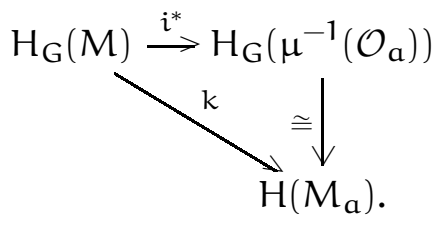

Here the vertical map is given by the inverse of the map

$$
\pi^{*}: \mathrm{H}\left(\mathrm{M}_{\mathrm{a}}\right) \rightarrow \mathrm{H}_{\mathrm{G}}\left(\mu^{-1}\left(\mathcal{O}_{\mathrm{a}}\right)\right), \quad[\eta] \mapsto\left[\pi^{*} \eta\right],
$$

which is an isomorphism due to Theorem 2.1.

If the generalized complex structure $\mathcal{J}$ on $M$ is induced by a symplectic structure, then it follows from the famous Kirwan surjectivity theorem [Kir86] that the Kirwan map is surjective. Let us give a very short explanation why the Kirwan surjectivity theorem holds in the symplectic case. For simplicity, let us only consider $S^{1}$ action here. In this case, the fixed points set of the action are exactly the critical points set of the moment map and the moment map is a Morse-Bott function. Besides, the norm square of the moment map induces a Morse stratification on the manifold $M$ from which one derives the Kirwan surjectivity theorem. However, for Hamiltonian $S^{1}$ action on general complex manifolds, in general the generalized moment map is not a Morse-Bott function. Indeed, let $\mu: M \rightarrow \mathbb{R}$ be the 
generalized moment map and $\alpha$ the moment one form. Then the condition $-X+\sqrt{-1}(d \mu+\sqrt{-1} \alpha) \in C^{\infty}(L)$ is equivalent to that

$$
\mathrm{J} d \mu=-X-\alpha,
$$

where $X$ is the vector field generated by the $S^{1}$ action, and $L$ is the $\sqrt{-1}$ eigenbundle of the generalized complex structure. Let $\operatorname{Crit}(\mu)$ be the set of critical points of the moment map $\mu$ and $M^{S^{1}}$ the fixed points set of the $S^{1}$ action. It is easy to see that

$$
\operatorname{Crit}(\mu) \subset M^{S^{1}}
$$

However, in general $\operatorname{Crit}(\mu) \neq M^{S^{1}}$. For instance, in [Hu05, Section 5] it was shown that there is a generalized Kähler structure $\left(\mathcal{J}_{1}, \mathcal{J}_{2}\right)$ on $\mathbb{C}^{2} \backslash\{0\}$ and a Hamiltonian $S^{1}$ action on $\left(\mathbb{C}^{2} \backslash\{0\}, \mathcal{J}_{1}, \mathcal{J}_{2}\right)$ such that for any regular value $a$ of the generalized moment map $\mu$, the level set $\mu^{-1}(a)$ contains a fixed points submanifold. It is unclear to the author at the moment whether the Kirwan surjectivity still holds or not for Hamiltonian actions on twisted generalized complex manifolds in general.

Nevertheless, the image of the Kirwan map forms a subgroup of the cohomology group of the generalized complex quotient which may contain many interesting cohomology classes. For instance, it is an interesting question when the generalized complex quotient has a non-trivial twisting. Observe that by Lemma 5.1 and Proposition 5.2 the cohomology class of the twisting three form $\widetilde{H}$ on $M_{a}$ is exactly the image of the equivariant cohomology class $[H+\alpha]$ under the Kirwan map $k: H_{G}(M) \rightarrow H\left(M_{a}\right)$. This innocuous observation leads to the following result.

Proposition 5.3. Assume that $(\mathrm{M}, \mathcal{J})$ is an $\mathrm{H}$-twisted generalized complex manifold such that $\mathrm{H}^{\text {odd }}(\mathrm{M})=0$. And assume there is a Hamiltonian action of a compact connected Lie group $\mathrm{G}$ on $\mathrm{M}$ with generalized moment map $\mu$ and moment one form $\alpha$. Let $\mathcal{O}_{\mathrm{a}}$ be a co-adjoint orbit through $\mathrm{a} \in \mathfrak{g}^{*}$ so that $\mathrm{G}$ acts freely on $\mu^{-1}\left(\mathcal{O}_{a}\right)$. Then the twisted three form $\widetilde{\mathrm{H}}$ on $\mathrm{M}_{\mathrm{a}}=\mu^{-1}\left(\mathcal{O}_{\mathrm{a}}\right) / \mathrm{G}$, as defined in Lemma 5.1, is cohomologically trivial.

Proof. It is shown in [GS99, Thm. 6.5.3] that if $\mathrm{H}^{\text {odd }}(M)=0$ then the spectral sequence of the Cartan complex of the G-manifold $M$ degenerates at the $E_{1}$ stage. In particular, we have that $\mathrm{H}_{\mathrm{G}}^{\text {odd }}(M) \cong \bigoplus_{\mathrm{p}+\mathrm{q}=\text { odd }} \mathrm{E}_{1}^{\mathrm{p}, \mathrm{q}}=$ $\mathrm{H}^{\text {odd }}(\mathrm{M}) \otimes\left(\mathrm{Sg}^{*}\right)^{\mathrm{G}}=0$. This shows clearly that $\mathrm{H}_{\mathrm{G}}(\mathrm{M})$ contains no nontrivial odd dimensional equivariant cohomology classes. We conclude that $[\mathrm{H}+\alpha]=0$ and so $[\widetilde{\mathrm{H}}]=\mathrm{k}([\mathrm{H}+\alpha])=0$.

Remark 5.4. Physicists are interested in producing explicit examples of gauged sigma models. When there is no flux, i.e., the target manifold does not have a cohomologically non-trivial twisting, a very useful tool producing such examples is to use the so called gauged linear model which 
corresponds to the Kähler quotient of a Kähler vector space. Naturally, physicists had hoped that one can produce explicit examples of generalized Kähler quotients of generalized Kähler vector spaces such that the quotient spaces have a cohomologically non-trivial twisting. The above result asserts that this is not possible since $H^{\text {odd }}(V)=0$ for any vector space $V$.

We finish this section by showing that in the framework of the twisted equivariant cohomology there is also a well-defined Kirwan map. This strongly suggests that the results we obtained in Section 4 do help us understand the cohomology of the generalized complex quotient. To describe the Kirwan map in this setting, we will need Proposition A.4 in the Appen$\operatorname{dix} \mathrm{A}$

Proposition 5.5. Assume a compact connected Lie group $\mathrm{G}$ acts on an $\mathrm{H}$-twisted generalized complex manifold $M$ with generalized moment map $\mu: M \rightarrow \mathfrak{g}^{*}$ and moment one form $\alpha \in \Omega^{1}\left(M, \mathfrak{g}^{*}\right)$. Assume that $\mathrm{G}$ acts freely on the level set $\mu^{-1}(0)$. Then there is a Kirwan map $\mathrm{k}: \mathrm{H}_{\mathrm{G}}(\mathrm{M}, \mathrm{H}+\alpha) \rightarrow \mathrm{H}\left(\mathrm{M}_{0}, \widetilde{\mathrm{H}}\right)$ such that the following diagram commutes:

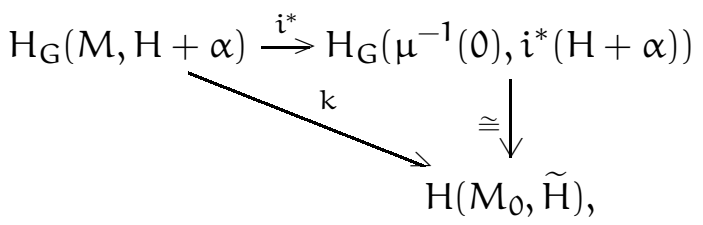

where $i^{*}: \mathrm{H}_{\mathrm{G}}(\mathrm{M}, \mathrm{H}+\alpha) \rightarrow \mathrm{H}_{\mathrm{G}}\left(\mu^{-1}(0), i^{*}(\mathrm{H}+\alpha)\right)$ is induced by the inclusion map $i: \mu^{-1}(0) \rightarrow M, M_{0}=\mu^{-1}(0) / G$ is the generalized complex quotient taken at the zero level of the generalized moment map, and $\widetilde{\mathrm{H}}$ is the twisting three form on $\mathrm{M}_{0}$ obtained as in Lemma 5.1 and Proposition 5.2

Proof. By Lemma 5.1 and Proposition 5.2, we can find a closed three form $\eta \in \Omega^{3}\left(\mu^{-1}(0)\right)$ such that $[\eta]=\left[i^{*}(H+\alpha)\right]$ and such that $\eta=\pi^{*} \widetilde{H}$. Since $\eta$ and $i^{*}(H+\alpha)$ represents the same cohomology class, we have

$$
\mathrm{H}_{\mathrm{G}}\left(\mu^{-1}(0), \eta\right) \cong \mathrm{H}_{\mathrm{G}}\left(\mu^{-1}(0), i^{*}(\mathrm{H}+\alpha)\right) \text {. }
$$

Corollary 5.5 now follows easily from Proposition A.4

\section{THE DUISTERMAAT-HECKMAN THEOREM FOR GENERALIZED CALABI-YAU MANIFOLDS}

Consider the Hamiltonian action of a compact connected Lie group G on an $\mathrm{H}$-twisted generalized Calabi-Yau manifold $M$. Our first observation is that if the action is free at a level set of the generalized moment map, then the generalized complex quotient taken at the level set inherits a generalized Calabi-Yau structure. The same result, under the assumption that both the twisting three form $\mathrm{H}$ and the moment one form vanish, has already been proved by Nitta [NY06]. We will make use of the following fact established in the proof of [NY06, Thm. A]. 
Lemma 6.1. Suppose that there is a Hamiltonian action of a compact connected Lie group $\mathrm{G}$ on an $\mathrm{H}$-twisted generalized Calabi-Yau manifold $\mathrm{M}$ with generalized moment map $\mu: M \rightarrow \mathfrak{g}^{*}$ and zero moment one form. Then the restriction of the generalized Calabi-Yau structure $\rho$ to $\mu^{-1}(0)$ is nowhere vanishing.

Remark 6.2. Although [NY06] assume the generalized Calabi-Yau structure is untwisted, the proof of the above fact given by Nitta uses only the linear algebra of generalized complex structures and so applies to the twisted case as well.

Proposition 6.3. Assume that there is a Hamiltonian action of a compact connected Lie group $\mathrm{G}$ on an $\mathrm{H}$-twisted generalized Calabi-Yau manifold $(\mathrm{M}, \mathcal{J})$ with generalized moment map $\mu: M \rightarrow \mathfrak{g}^{*}$ and moment one form $\alpha \in \Omega^{1}\left(M, \mathfrak{g}^{*}\right)$, preserving the generalized Calabi-Yau structure $\rho$. And assume $\mathcal{O}_{\mathrm{a}}$ is a co-adjoint orbit through $\mathrm{a} \in \mathfrak{g}^{*}$ so that $\mathrm{G}$ acts freely on $\mu^{-1}\left(\mathcal{O}_{\mathrm{a}}\right)$. Then given a connection on $\mu^{-1}\left(\mathcal{O}_{a}\right)$, the generalized complex quotient $M_{a}$ inherits an $\widetilde{\mathrm{H}}$-twisted generalized Calabi-Yau structure $\widetilde{\rho}$, such that $\pi^{*} \widetilde{\rho}=\left.\rho\right|_{\mu^{-1}\left(\mathcal{O}_{a}\right)}$, where $\pi: \mu^{-1}\left(\mathcal{O}_{a}\right) \rightarrow M_{a}$ is the quotient map, $\widetilde{\mathrm{H}}$ is defined as in Lemma 5.1. Up to B-transform, $\widetilde{\rho}$ is independent of the choice of connections. Finally, for all $\mathrm{m} \in \mu^{-1}\left(\mathcal{O}_{\mathrm{a}}\right)$,

$$
\operatorname{type}(\tilde{\mathcal{J}})_{[\mathrm{m}]}=\operatorname{type}(\mathcal{J})_{\mathrm{m}}
$$

where $\widetilde{\mathrm{J}}$ is the quotient generalized complex structure on $\mathrm{M}_{\mathrm{a}}$.

Proof. It suffices to show that the restriction of $\rho$ to $\mu^{-1}\left(\mathcal{O}_{a}\right)$ descends to a generalized Calabi-Yau structure $\widetilde{\rho}$ on $M_{a}$ such that $\pi^{*} \widetilde{\rho}=\left.\rho\right|_{\mu^{-1}\left(\mathcal{O}_{a}\right)}$. Using the argument given in the proof of [LT05, Prop. 3.8, A.7], without the loss of generality, we can assume that the coadjoint orbit $\mathcal{O}_{a}=0$, and assume that in an open neighborhood of the level set $\mu^{-1}(0)$, the moment one form is zero and the twisting three form $\mathrm{H}$ is basic so that it descends to the twisting three form $\widetilde{H}$ on the quotient $M_{0}:=\mu^{-1}(0) / G$. By the definition of the generalized moment map, we have that for any $\xi \in \mathfrak{g}, \xi_{M}-\sqrt{-1} \mathrm{~d} \mu^{\xi} \in C^{\infty}(\mathrm{L})$, where $L$ is the $\sqrt{-1}$ eigenbundle of the generalized complex structure $\mathcal{J}$. By the definition of a generalized Calabi-Yau structure, we have

$$
\left(\xi_{M}-\sqrt{-1} \mathrm{~d} \mu^{\xi}\right) \cdot \rho=\iota_{\xi_{M}} \rho-\sqrt{-1} \mathrm{~d} \mu^{\xi} \wedge \rho=0 .
$$

It follows that $\left.\iota_{\xi_{M}} \rho\right|_{\mu^{-1}(0)}=\left.\left(\sqrt{-1} d \mu^{\xi} \wedge \rho\right)\right|_{\mu^{-1}(0)}=0$ for any $\xi \in \mathfrak{g}$. Therefore $\left.\rho\right|_{f^{-1}(0)}$ is a basic form and so descends to a form $\widetilde{\rho}$ such that $\pi^{*} \widetilde{\rho}=\left.\rho\right|_{\mu^{-1}(0)}$. Since $\rho$ is $d_{H^{-c l o s e d}}, \widetilde{\rho}$ is $d_{\widetilde{H}^{-c l o s e d}}$; moreover, since by Lemma $\left.6.1 \rho\right|_{\mu^{-1}(0)}$ is nowhere vanishing, $\widetilde{\rho}$ is nowhere vanishing as well. Thus

$$
\mathrm{L}_{\widetilde{\rho}}(x)=\left\{A \in \mathrm{T}_{\mathbb{C}, x} M_{0} \oplus \mathrm{T}_{\mathbb{C}, x}^{*} M_{0} \mid A \cdot \widetilde{\rho}=0\right\}
$$

is an isotropic subspace of $\mathrm{T}_{\mathbb{C}, x} M_{0} \oplus \mathrm{T}_{\mathbb{C}, x}^{*} M_{0}, x \in M_{0}$. Finally, let $\widetilde{L}$ be the $\sqrt{-1}$ eigenbundle of the quotient generalized complex structure on $M_{0}$. Using the description of $\widetilde{L}$ in [LT05, Prop. 3.8] it is straightforward to check 
that $\widetilde{\rho}$ is annihilated by the Clifford action of the sections of $\widetilde{L}$. Hence we have $\widetilde{\mathrm{L}}_{x} \subset \mathrm{L}_{\tilde{\rho}}(x), x \in M_{0}$. However, $\mathrm{L}_{x}$ is a maximal isotropic subspace of $\mathrm{T}_{\mathbb{C}, x} \mathrm{M}_{0} \oplus \mathrm{T}_{\mathbb{C}, x}^{*} \mathrm{M}_{0}$. So we must have $\widetilde{\mathrm{L}}_{x}=\mathrm{L}_{\widetilde{\rho}}(x), x \in M$. In other words, $\widetilde{\rho}$ is the pure spinor associated to the quotient generalized complex structure $\widetilde{J}$ on $M_{0}$. This finishes the proof that $\widetilde{\rho}$ is a generalized Calabi-Yau structure.

Example 6.4. Let $\mathrm{G}$ act on a symplectic manifold $(M, \omega)$ with moment map $\Phi: M \rightarrow \mathfrak{g}^{*}$, that is, $\Phi$ is equivariant and $\iota_{\xi_{M}} \omega=-\mathrm{d} \Phi^{\xi}$ for all $\xi \in \mathfrak{g}$. Then the action of $\mathrm{G}$ also preserves the generalized complex structure $\mathcal{J}_{\omega}$, where $\mathcal{J}_{\omega}$ is defined as in Example 3.4, and it is straightforward to check that the action on the generalized complex manifold $\left(M, \mathcal{J}_{\omega}\right)$ is Hamiltonian with generalized moment map $\Phi$ and trivial moment one form. By Example $3.4 \omega$ defines a generalized Calabi-Yau structure $e^{i \omega}$. Suppose that $G$ acts freely on the level set $\mu^{-1}(0)$. Then the restriction of $e^{i \omega}$ to the level set $\mu^{-1}(0)$ descends to the quotient generalized Calabi-Yau structure $e^{i \omega_{0}}$ on $M_{0}=\mu^{-1}(0) / G$, where $\omega_{0}$ is the reduced symplectic form on $M_{0}$.

On the generalized complex quotient $M_{a}$, the quotient generalized CalabiYau structure $\widetilde{\rho}_{a}$ depends on the choice of a connection, c.f., [LT05, Prop. A.7]. However, a different choice of the connection results in a new quotient generalized Calabi-Yau structure which is given by $e^{-B} \widetilde{\rho}_{a}$ for some closed two form $B \in \Omega^{2}\left(M_{a}\right)$. It follows immediately from Lemma 3.1 that, on the quotient $M_{a},\left(\widetilde{\rho}_{a}, \overline{\widetilde{\rho}}_{a}\right)$ is well-defined, independent of the choice of connections, where $(\cdot, \cdot)$ denotes the Mukai pairing on the quotient space $\mathrm{M}_{\mathrm{a}}$.

From now on we consider the Hamiltonian action of a compact connected torus $T$ on an $H$-twisted generalized complex manifold $M$ with a proper generalized moment map $\mu$. Assume that $a_{0} \in \mathfrak{t}^{*}$ such that the action of $T$ on the level set $\mu^{-1}\left(a_{0}\right)$ is free. Since by Lemma 6.5 below the set on which $T$ acts freely is open and since the generalized moment map is proper, there is an open subset $\mathrm{U} \subset \mathfrak{t}^{*}$ such that for any $\mathrm{a} \in \mathrm{U}$ the action of $T$ on $\mu^{-1}(a)$ is free. We define the Duistermatt-Heckman function $f$ on $\mathrm{U} \subset \mathfrak{t}^{*}$ by the following formula.

$$
f(a)=\frac{(-1)^{n+\frac{k(k+1)}{2}}(2 \pi)^{k}}{(2 i)^{n-k}} \int_{M_{a}}\left(\widetilde{\rho}_{a}, \overline{\widetilde{\rho}}_{a}\right),
$$

where $\widetilde{\rho}_{a}$ is the quotient generalized Calabi-Yau structure on $M_{a}$, and $M_{a}$ is given the orientation induced by $\left(\widetilde{\rho}_{a}, \overline{\widetilde{\rho}}_{a}\right)$.

Lemma 6.5. ([GS84] ) Assume that a torus $\mathrm{T}$ acts on a connected manifold $M$ effectively. Then the set $\mathrm{M}^{\prime}$ on which $\mathrm{T}$ acts freely is equal to the complement of a locally finite union of closed manifolds of codimension $\geq 2$. In particular, $M^{\prime}$ is open, connected, dense, and $M \backslash M^{\prime}$ has measure zero. 
Theorem 6.6. Suppose that a $\mathrm{k}$ dimensional torus $\mathrm{T}$ acts on a $2 \mathrm{n}$ dimensional connected generalized Calabi-Yau manifold $M$ freely such that the action is Hamiltonian with a proper generalized moment map $\mu: M \rightarrow t^{*}$ and a moment one form $\alpha \in \Omega^{1}\left(M, t^{*}\right)$, and such that the action preserves the generalized Calabi-Yau structure $\rho$ on $M$. Then the density function of the push-forward measure $\mu_{*}(\mathrm{dm})$ coincides with the Duistermaat-Heckman function $\mathrm{f}$ defined by (6.1), where $\mathrm{dm}$ is the measure on $\mathrm{M}$ defined by the volume form (3.4) on $\mathrm{M}$.

Proof. Since the torus action is free, after applying a B-transform, we may well assume that the moment one form $\alpha$ is trivial. Choose an integer lattice in $\mathfrak{t}=\operatorname{Lie}(T)$, and identify $T$ with $S^{1} \times \underbrace{S^{1} \cdots \times S^{1}}_{k-1}=S^{1} \times T^{k-1}$. Following the proof of [GGK97, Thm. 5.8], we consider an open neighborhood of a free orbit in $M$. On such a neighborhood there exists a coordinate system

$$
\theta_{1}, \theta_{2}, \cdots, \theta_{k}, x_{1}, \cdots, x_{k}, y_{1}, \cdots, y_{2 d} \text {, }
$$

where $2 d=2 n-2 k, \theta_{i} \in \mathbb{R} / 2 \pi \mathbf{Z}$, and $x_{i}$ are coordinates on $\mathfrak{t}^{*}$, such that the T action is generated by the vector field $\frac{\partial}{\partial \theta_{1}}, \frac{\partial}{\partial \theta_{2}}, \cdots, \frac{\partial}{\partial \theta_{k}}$ and the moment map $\mu$ is given by $\left(x_{1}, x_{2}, \cdots, x_{k}\right)$. We refer to the proof of [GGK97, Thm. 5.8] for a detailed explanation on the existence of such a coordinate system. Then we claim that for any $a=\left(a_{1}, a_{2}, \cdots, a_{k}\right)$ in an open subset of $t^{*}$ such that $x_{1}, x_{2}, \cdots, x_{k}$ are well defined, we have

$$
(\rho(x), \bar{\rho}(x))_{M}=(-2 i)^{k}\left(\theta_{1} \wedge d x_{1} \wedge \theta_{2} \wedge d x_{2} \wedge \cdots \wedge \theta_{k} \wedge d x_{k}\right) \wedge\left(\widetilde{\rho}_{a}(\pi(x)), \widetilde{\widetilde{\rho}}_{a}(\pi(x))\right)_{M_{a}},
$$

where $\pi: \mu^{-1}(a) \rightarrow M_{a}$ is the quotient map, $x$ is a point in the level set $\mu^{-1}(a)$, and $(\cdot, \cdot)_{M}$ and $(\cdot, \cdot)_{M_{a}}$ denote the Mukai pairing on $M$ and $M_{a}$ respectively. We will establish our claim by induction on $k$. If $k=1$, then since the action is Hamiltonian, $\left(\frac{\partial}{\partial \theta_{1}}-\sqrt{-1} d x_{1}\right) \cdot \rho=0$, i.e., $\iota \frac{\partial}{\partial \theta_{1}} \rho=$ $\sqrt{-1} \mathrm{~d} x_{1} \wedge \rho$. A simple calculation shows that under the above coordinate system $\rho$ must be of the form

$$
\mathrm{d} x_{1} \wedge \alpha_{0}+i \theta_{1} \wedge \mathrm{d} x_{1} \wedge \alpha_{1}+\alpha_{1},
$$

where $\alpha_{i}$ is a differential form such that $\iota_{\frac{\partial}{\partial \theta_{1}}} \alpha_{i}=0$ and $\iota_{\frac{\partial}{\partial x_{1}}} \alpha_{i}=0, i=0,1$. It is easy to check directly that the restriction of $\alpha_{1}$ to the level set $x_{1}^{-1}(a)$ descends to a quotient Calabi-Yau structure $\widetilde{\alpha}_{1}$ on the quotient $x_{1}^{-1}\left(a_{1}\right) / S^{1}$; moreover, we have

$$
(\rho(x), \bar{\rho}(x))_{M}=-2 i\left(\theta_{1} \wedge d x_{1}\right) \wedge\left(\widetilde{\alpha}_{1}\left(\pi_{1}(x)\right), \overline{\widetilde{\alpha}}_{1}\left(\pi_{1}(x)\right)\right)_{M_{a_{1}}},
$$

where $\pi_{1}: x_{1}^{-1}\left(a_{1}\right) \rightarrow M_{a_{1}}=x_{1}^{-1}\left(a_{1}\right) / S^{1}$ is the quotient map. If $k=1$, then $M_{a_{1}}=M_{a}$ and $\widetilde{\alpha}_{1}$ is actually the quotient generalized Calabi-Yau structure $\widetilde{\rho}_{a}$ on it. The claim is proved for the case $k=1$. Assume that the claim is true for $k-1$ and consider the case $k$. Note that the action of $\mathrm{T}^{\mathrm{k}-1} \subset \mathrm{T}=\mathrm{S}^{1} \times \mathrm{T}^{\mathrm{k}-1}$ on $\mathrm{M}$ commutes with that of $\mathrm{S}^{1}$ and so descends 
to a Hamiltonian action on $M_{a_{1}}$ with moment map $\left(x_{2}, \cdots, x_{k}\right)$. Here by abuse of notation, we have used the same $x_{i}$ to denote the functions on the quotient $M_{a_{1}}$ whose pull-back under the quotient map $\pi_{1}$ coincides with $\left.x_{i}\right|_{x_{1}^{-1}\left(a_{1}\right)}, 2 \leq i \leq k$.

Observe that $M_{a}=\left(x_{2}^{-1}\left(a_{2}\right) \cap \cdots x_{k}^{-1}\left(a_{k}\right)\right) / T^{k-1}$ and the restriction of $\widetilde{\alpha}_{1}$ to $x_{2}^{-1}\left(a_{2}\right) \cap \cdots x_{k}^{-1}\left(a_{k}\right)$ descends to the quotient generalized Calabi-Yau structure $\widetilde{\rho}_{a}$ on $M_{a}$. Using the induction assumption, we get that

$$
\left(\widetilde{\alpha}_{1}(y), \overline{\widetilde{\alpha}}_{1}(y)\right)_{M_{a_{1}}}=(-2 i)^{k-1}\left(\theta_{2} \wedge d x_{2} \wedge \cdots \wedge \theta_{k} \wedge d x_{k}\right) \wedge\left(\widetilde{\rho}_{a}\left(\pi_{2}(y)\right), \overline{\tilde{\rho}}_{a}\left(\pi_{2}(y)\right)\right)_{M_{a}},
$$

where $\pi_{2}: x_{2}^{-1}\left(a_{2}\right) \cap \cdots x_{k}^{-1}\left(a_{k}\right) \rightarrow M_{a}=\left(x_{2}^{-1}\left(a_{2}\right) \cap \cdots x_{k}^{-1}\left(a_{k}\right)\right) / T^{k-1}$ is the quotient map, $y$ is a point in the level set $x_{2}^{-1}\left(a_{2}\right) \cap \cdots x_{k}^{-1}\left(a_{k}\right)$. Combining (6.3) and (6.4), we conclude our claim holds for the case $k$.

To finish the proof, we borrow the following argument from [GGK97]. Let $\lambda_{j}$ be an invariant partition of unit such that each $\lambda_{j}$ is supported in a neighborhood with coordinates described above. Then we have

$$
\begin{aligned}
\mu_{*}(\mathrm{dm}) & =\frac{(-1)^{n}}{(2 i)^{n}} \sum_{j} \int_{M} \lambda_{j}(\rho(x), \bar{\rho}(x))_{M} \\
& =\frac{(-1)^{n-k}}{(2 i)^{n-k}} \sum_{j} \int \lambda_{j}\left(\theta_{1} \wedge d x_{1} \wedge \cdots \wedge \theta_{k} \wedge d x_{k}\right) \wedge\left(\widetilde{\rho}_{a}(\pi(x)), \overline{\widetilde{\rho}}_{a}(\pi(x))\right)_{M_{a}} \\
& =\frac{(-1)^{n+\frac{k(k+1)}{2}}(2 \pi)^{k}}{(2 i)^{n-k}} \sum_{j} \int \lambda_{j}\left(\int_{M_{a}}\left(\widetilde{\rho}_{a}(\pi(x)), \overline{\widetilde{\rho}}_{a}(\pi(x))\right)_{M_{a}}\right) d_{1} d x_{2} \cdots d x_{k}
\end{aligned}
$$

( Because of Fubini's theorem)

$$
\begin{aligned}
& =\frac{(-1)^{n+\frac{k(k+1)}{2}}(2 \pi)^{k}}{(2 i)^{n-k}} \int \sum_{j} \lambda_{j}\left(\int_{M_{a}}\left(\widetilde{\rho}_{a}(\pi(x)), \overline{\widetilde{\rho}}_{a}(\pi(x))\right)_{M_{a}}\right) d x_{1} d x_{2} \cdots d x_{k} \\
& =\int f(a) d x_{1} d x_{2} \cdots d x_{k} .
\end{aligned}
$$

This proves Theorem 6.6 .

Before we state the Duistermaat-Heckman theorem in the generalized Calabi-Yau setting, we first give two simple observations.

Lemma 6.7. Let $\sigma$ be the anti-automorphism defined on $\mathrm{C}^{\infty}\left(\wedge \mathrm{T}^{*} \mathrm{M}\right)$ as in (3.1), and let $\mathrm{H}$ be a closed thee form on the manifold $\mathrm{M}$. We have

a) if a differential form $\varphi$ is $\mathrm{d}_{\mathrm{H}}=(\mathrm{d}-\mathrm{H} \wedge)$-closed, then $\sigma(\varphi)$ is $\mathrm{d}_{-\mathrm{H}}=$ $(\mathrm{d}+\mathrm{H} \wedge)$-closed;

b) if $\mathrm{X}+\gamma \in \mathrm{C}^{\infty}\left(\mathrm{TM} \oplus \mathrm{T}^{*} \mathrm{M}\right)$ and if $(\mathrm{X}+\gamma) \cdot \varphi=\mathrm{\imath} \mathrm{X} \gamma+\gamma \wedge \varphi=0$, then we have $(X-\gamma) \cdot \sigma(\varphi)=\iota_{X} \sigma(\varphi)-\gamma \wedge \sigma(\varphi)=0$.

Proof. Decompose $\varphi=\sum_{i=0}^{p} \varphi_{i}$ into homogeneous components by the usual grading of differential forms, where $p=\operatorname{dim} M$. Then it follows from 
$\mathrm{d}_{H} \varphi=0$ that $\mathrm{d} \varphi_{i}=0$ for $i \leq 1$ and $\mathrm{d} \varphi_{i}=\mathrm{H} \wedge \varphi_{i-2}$ for $i \geq 2$. It is straightforward to check that $\operatorname{d} \sigma\left(\varphi_{i}\right)=0$ if $i \leq 1$ and $\operatorname{d} \sigma\left(\varphi_{i}\right)=-\mathrm{H} \wedge \sigma\left(\varphi_{i-2}\right)$ if $i \geq 2$. So we have that $\sigma(\varphi)$ is $d_{-H}=(d+H \wedge)$-closed. This proves Assertion (a) in Lemma 6.7. A similar argument proves Assertion (b).

Lemma 6.8. Assume that there is a Hamiltonian action of a compact connected Lie group $\mathrm{G}$ on an $\mathrm{H}$-twisted generalized Calabi-Yau manifold $\mathrm{M}$ with proper generalized moment map $\mu: M \rightarrow \mathfrak{t}^{*}$ and moment one form $\alpha \in \Omega^{1}\left(M, \mathfrak{g}^{*}\right)$. Then $e^{i \mu} \rho$ is an $\mathrm{H}_{\mathrm{G}}=(\mathrm{H}+\alpha)$-twisted equivariantly closed form with coefficients in the ring of formal power series.

Proof. For any $\xi \in \mathfrak{t}$, we have

$$
\begin{aligned}
\left(d_{G, H_{G}} e^{i \mu} \rho\right)(\xi) & =\left(\sqrt{-1} d u^{\xi} \wedge \rho-\iota_{\xi_{M}} \rho-\alpha^{\xi} \wedge \rho\right) e^{i \mu^{\xi}}+e^{i \mu}\left(d_{H} \rho\right) \\
& =\left(-\xi_{M}+\sqrt{-1}\left(d \mu^{\xi}+\sqrt{-1} \alpha^{\xi}\right)\right) \cdot \rho \\
& =0 .
\end{aligned}
$$

The last equality holds because $-\xi_{M}+\sqrt{-1}\left(d \mu^{\xi}+\sqrt{-1} \alpha^{\xi}\right)$ is a section of the $\sqrt{-1}$-eigenbundle of the generalized Calabi-Yau structure so that its Clifford action annihilates $\rho$.

Remark 6.9. The same observation that $e^{i \mu} \rho$ is an equivariant closed extension of $\rho$ has been made in [HuUribe06], and in [NY07] for the case $\mathrm{H}=0$ and $\alpha=0$. However, $e^{i \mu} \rho$ was treated as an equivariant differential form in the usual Cartan model in [NY07]. This is incorrect since $e^{i \mu} \rho: \mathfrak{g} \rightarrow \Omega(M)$ is not a polynomial map, where $\mathfrak{g}$ is the Lie algebra of $G$. For instance, let G act on a symplectic manifold $(M, \omega)$ with moment map $\Phi: M \rightarrow \mathfrak{g}^{*}$, and let $\rho=e^{i \omega}$. Then $e^{i \Phi} e^{i \omega}=e^{i(\omega+\Phi)}=e^{i \omega_{G}}$, where $\omega_{G}:=\omega+\Phi$ is the equivariant symplectic form. It is well known that $e^{i \omega_{G}}$ is not an equivariant differential form in the Cartan model, c.f., [GS99, pp. 167]. Therefore, strictly speaking, the proof of the Duistermaat-Heckman theorem in the generalized Calabi-Yau setting given in [NY07] has a gap.

Theorem 6.10. Assume that there is a free Hamiltonian action of a $\mathrm{k}$ dimensional torus $\mathrm{T}$ on an $\mathrm{H}$-twisted $2 \mathrm{n}$ dimensional generalized Calabi-Yau manifold $M$ with proper generalized moment map $\mu: M \rightarrow t^{*}$ and moment one form $\alpha \in \Omega^{1}\left(M, t^{*}\right)$, preserving the generalized Calabi-Yau structure $\rho$. Then the density function $\mathrm{f}$ for the push-forward measure on $\mathrm{t}^{*}$ via the generalized moment map $\mu$ is a polynomial of degree at most $n-k$.

Proof. As the action is free, after applying a B-transform, one can assume that the moment one form $\alpha$ is zero. Under this assumption, $\mathrm{H}_{\mathrm{G}}=\mathrm{H}$ is an equivariantly closed three form in the usual Cartan model. First, by Lemma 6.8 we have $d_{G, H}\left(e^{i \mu} \rho\right)=0$. Since $d_{G, H}$ is a real operator, we also have $d_{G, H}\left(e^{-i \mu} \bar{\rho}\right)=0$. Next we observe that the canonical antiautomorphism $\sigma: \Omega^{*}(M) \rightarrow \Omega^{*}(M)$ extends naturally to the equivariant 
differential forms. Moreover, as an easy consequence of Lemma 6.7, we have that

$$
d_{G,-H}\left(e^{-i \mu} \sigma(\rho)\right)=e^{-i \mu}(d+H \wedge) \sigma(\rho)+e^{-i \mu}\left(-\sqrt{-1} d \mu^{\xi}-\xi_{M}\right) \cdot \sigma(\rho)=0
$$

Consequently by Lemma $2.3 e^{-2 i \mu} \sigma(\rho) \wedge \bar{\rho}$ is equivariantly closed under the usual (untwisted) equivariant differential $d_{G}$.

Now choose a T-invariant $t^{*}$ valued connection one form $\theta$ for the principal bundle $M \rightarrow M / T$. Choose a basis of $\mathfrak{t}$ so as to identify $\mathfrak{t}^{*}$ with $\mathbb{R}^{k}$ and suppose $\theta=\left(\theta^{1}, \theta^{2}, \cdots, \theta^{k}\right), \mu=\left(\mu^{1}, \mu^{2}, \cdots, \mu^{k}\right)$ under this identification. Let $c_{l}$ be the unique closed two form on $M / T$ such that $\pi^{*} c_{l}=d \theta^{l}$, $1 \leq \mathrm{l} \leq \mathrm{k}$. As we explained in Appendix A the usual Cartan map, as defined in (2.3), extends naturally to the equivariant differential forms with coefficients in the ring of formal power series. Moreover, it follows easily from Theorem $\mathrm{A} .3$ that $e^{-2 i \mu} \sigma(\rho) \wedge \bar{\rho}$ gets mapped to an ordinary closed differential form

$$
e^{-2 i \mu^{l} c_{l}} \sigma(\tau) \wedge \bar{\tau}
$$

where $\tau$ is the unique differential form on $M / T$ such that $\pi^{*} \tau=\rho_{\text {hor }}$, and $\pi: M \rightarrow M / T$ is the quotient map.

Let $j_{a}: M_{a} \rightarrow M / T$ be the inclusion map and let $\pi_{a}: \mu^{-1}(a) \rightarrow M_{a}$ be the natural projection map. From the commutative diagram

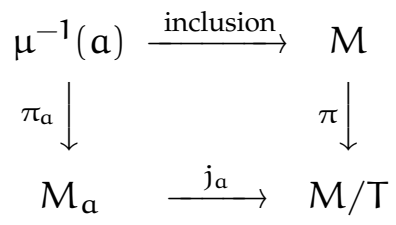

we get that $j_{a}^{*} \tau=\widetilde{\rho}$. The closed three form $H$ descends to a closed three form $\widetilde{\mathrm{H}}$ on $M / T$ such that $\pi^{*} \widetilde{\mathrm{H}}=\mathrm{H}$ and such that the quotient generalized Calabi-Yau structure $\rho_{a}$ is $d_{\widetilde{H}^{-c l o s e d}}$. By Lemma 6.7 $\sigma\left(\rho_{a}\right)$ is $d_{-\widetilde{H}^{-}}$ closed. Note that $d_{\widetilde{H}}$ is a real operator and $\overline{\widetilde{\rho}}$ is $d_{\widetilde{H}}$-closed. Hence $\sigma\left(\rho_{a}\right) \wedge \rho$ is an ordinary closed differential form, representing a cohomology class in $H(M / T, \mathbb{C})$. Now let $\varphi$ be the cohomology class of $\left[e^{-2 i \mu^{l} c_{l}} \sigma(\tau) \wedge \bar{\tau}\right]$ in $\mathrm{H}(\mathrm{M} / \mathrm{T}, \mathbb{C})$. Then we have

$$
\begin{aligned}
{\left[\sigma\left(\widetilde{\rho}_{a}\right) \wedge \overline{\widetilde{\rho}}_{a}\right] } & =\left[j_{a}^{*}(\sigma(\tau) \wedge \bar{\tau})\right] \\
& =\left[j_{a}^{*}\left(e^{2 i \mu^{l} c_{l}} \wedge\left(e^{-2 i \mu^{l} c_{l}} \wedge \sigma(\tau) \wedge \bar{\tau}\right)\right)\right] \\
& =\left[e^{2 i a^{l} \widetilde{c}_{l}} \wedge j_{a}^{*}\left(e^{-2 i \mu^{l} c_{l}} \wedge \sigma(\tau) \wedge \bar{\tau}\right)\right] \\
& =\left[e^{2 i a^{l} \widetilde{c}_{l}}\right] \wedge j_{a}^{*} \varphi,
\end{aligned}
$$

where $\rho_{a}$ is the quotient generalized Calabi-Yau structure on $M_{a}$, and $\widetilde{c}_{l}=$ $j_{a}^{*} c_{l}$. Since $M_{a}$ is compact and oriented, the embedding $j_{a}: M_{a} \rightarrow M / T$ defines an integral homology class $\left[M_{a}\right] \in H_{2 n-2 k}(M / T, Z)$. This homology 
class depends smoothly on a and is an integral class. So it is actually independent of $a$. Let us fix an $a_{0}$ in the moment map image $\mu(M)$. Then we have $\left[M_{a_{0}}\right]=\left[M_{a}\right]$. Thus up to a normalization factor the integral (6.1) can be interpreted topologically as the pairing of the constant homology class $\left[\mathrm{M}_{\mathrm{a}_{0}}\right]$ with the cohomology class

$$
\left[e^{2 i a^{l} \widetilde{c}_{l}}\right] \wedge j_{a}^{*} \varphi .
$$

Note that $\widetilde{c}_{l}$ represents a degree two cohomology class, $1 \leq l \leq k$. Therefore the density function (6.1) is a polynomial function of degree at most $n-k$.

Remark 6.11. If the generalized Calabi-Yau structure $\rho$ is of constant type $p$, then by the generalized Darboux theorem locally it can always be written as $e^{B+i \omega_{\theta}} \theta_{1} \wedge \cdots \wedge \theta_{p}$, where $B+i \omega$ is a closed complex valued two form, and $\theta_{1}, \cdots, \theta_{p}$ are complex valued one forms. (We refer to [Gua07, Thm. 4.35] for details.) Combining this with (6.5), we conclude that the DuistermaatHeckman function is of degree at most $n-k-p$ provided the generalized Calabi-Yau structure $\rho$ is of constant type $p$.

\section{EXAMPLES OF HAMILTONIAN ACTIONS ON GENERALIZED CALABI-YAU MANIFOLDS}

The following theorem extends a useful construction of Hamiltonian actions in symplectic geometry [L04, Prop. 4.2] to generalized complex geometry, and it is an equivariant version of a construction proposed in [Ca05. Thm. 2.2]. It allows us to construct examples of Hamiltonian actions on compact twisted generalized Calabi-Yau manifolds which are not a direct product of a symplectic manifold and a complex manifold.

Theorem 7.1. Let $(\mathrm{N}, \mathcal{J})$ be a compact $\mathrm{H}$-twisted generalized Calabi-Yau manifold. Then there exists a $\mathrm{S}^{2}$ bundle $\pi: M \rightarrow \mathrm{N}$ which satisfies

a) $M$ admits a $\pi^{*} \mathrm{H}$-twisted generalized Calabi-Yau structure $\mathcal{J}^{\prime}$;

b) there exists a Hamiltonian $S^{1}$ action on the generalized Calabi-Yau manifold $\left(M, \mathcal{J}^{\prime}\right)$.

Proof. Let $S^{2}$ be the set of unit vectors in $\mathbb{R}^{3}$. In cylindrical coordinates $(\theta, h)$ away from the poles, $0 \leq \theta<2 \pi,-1 \leq h \leq 1$, the standard symplectic form on $S^{2}$ is the area form $\sigma=d \theta \wedge d h$. The circle $S^{1}$ acts on $S^{2}$ by rotations

$$
e^{i t}(\theta, h)=(\theta+t, h) \text {. }
$$

This action is Hamiltonian with the moment map given by $\mu=h$, i.e., the height function.

Let $\pi_{\mathrm{P}}: \mathrm{P} \rightarrow \mathrm{N}$ be the principal $\mathrm{S}^{1}$-bundle with Euler class $[c] \in \mathrm{H}^{2}(\mathrm{~N}, \mathbf{Z})$, let $\Theta$ be the connection 1-form such that $\pi_{\mathrm{P}}^{*} \mathrm{c}=\mathrm{d} \Theta$, and let $M$ be the associated bundle $P \times{ }_{S^{1}} S^{2}$. Then $\pi: M \rightarrow N$ is a symplectic fibration over the compact $\mathrm{H}$-twisted generalized complex manifold $\mathrm{N}$. The standard symplectic form $\sigma$ on $S^{2}$ gives rise to a symplectic form $\sigma_{x}$ on each fibre 
$\pi^{-1}(x) \cong S^{2}$, where $x \in N$, whereas the height function $h$ on $S^{2}$ gives rise to a global function $F$ on $M$ whose restriction to each fiber is just $h$.

Next we resort to minimal coupling construction to get a closed two form $\eta$ which restricts to $\sigma_{x}$ on each fiber $\pi^{-1}(x)$. Let us give a sketch of this construction here and refer to [GS84] for technical details. Consider the closed tow form $-d(t \Theta)=-t d \Theta-d t \wedge \Theta$ defined on $P \times \mathbb{R}$. It is easy to check that the $S^{1}$ action defined by

$$
e^{i \theta}(p, t)=\left(e^{i \theta} p, t\right)
$$

is Hamiltonian with moment map $t$. Thus the diagonal action of $S^{1}$ on $(P \times$ $\mathbb{R}) \times S^{2}$ is also Hamiltonian, and $M$ is just the reduced space of $(P \times \mathbb{R}) \times S^{2}$ at the zero level. As a result, the closed two form $\left.(-\mathrm{d}(\mathrm{t} \Theta)+\sigma)\right|_{\text {zero level }}$ descends to a closed two form $\eta$ on $M$ which restricts to $\sigma_{x}$ on each fibre $\pi^{-1}(x) \cong S^{2}$, and the function

$$
(\mathrm{P} \times \mathbb{R}) \times \mathrm{S}^{2} \rightarrow \mathbb{R},(\mathrm{p}, \mathrm{t}, z) \mapsto \mathrm{h}(z)
$$

descends to a function $F$ on $M$ which restricts to the height function $h$ on each fibre $S^{2}$. Moreover, there is another $S^{1}$ action on $(P \times \mathbb{R}) \times S^{2}$ which is defined by letting $S^{1}$ act on $(P \times \mathbb{R})$ trivially and act on $S^{2}$ by rotation; this action commutes with the above diagonal $S^{1}$ action and so descends to a fibrewise $S^{1}$ action on $M$. Let $X$ be the fundamental vector field generated by this fibrewise $S^{1}$ action. It is easy to check that the function $F$ is invariant under the fibrewise $S^{1}$ action and $\iota_{X}(\eta)=d F$.

Let $\rho$ be the closed nowhere vanishing pure spinor associated to the generalized Calabi-Yau structure $\mathcal{J}$ on $N$, and let $(\cdot, \cdot)_{M}$ be the Mukai pairing on $M$. We claim that for sufficiently small $\epsilon>0$,

$$
\left(e^{i \epsilon \eta} \wedge \pi^{*} \rho, e^{-i \epsilon \eta} \wedge \pi^{*} \bar{\rho}\right)_{M} \neq 0 .
$$

Since $\eta$ is non-degenerate on the vertical tangent space $\operatorname{ker} \pi_{*}$, at each point $x \in M$ it determines a horizontal subspace

$$
\text { Hor }_{x}=\left\{X \in T_{\chi} M: \eta(X, Y)=0, \forall Y \in T_{\chi} S^{2}\right\} .
$$

The subspace Hor ${ }_{x}$ is a complement to $T_{\chi} S^{2}$ and is isomorphic to $T_{\pi(x)} N$ via $\pi_{*}$. So we have a spitting

$$
\mathrm{T}_{x} M=\operatorname{Hor}_{x} \oplus \mathrm{T}_{\mathrm{x}} \mathrm{S}^{2}
$$

which further induces a splitting of the space of differential forms of degree two

$$
\wedge^{2} \mathrm{~T}_{x}^{*} \mathrm{M}=\left(\wedge^{2} \operatorname{Hor}_{\mathrm{x}}^{*}\right) \oplus\left(\wedge^{2} \mathrm{~T}_{\chi}^{*} \mathrm{~S}^{2}\right) \oplus\left(\mathrm{T}_{\mathrm{x}}^{*} \mathrm{M} \otimes \text { Hor }_{\mathrm{x}}^{*}\right) .
$$

Here Hor* denotes the dual space of Hor $_{x}$. By the definition of the horizontal subspaces, $\eta$ splits into a direct sum $\eta=\eta_{1}+\eta_{2}$ such that $\eta_{1, x} \in$ $\wedge^{2} \operatorname{Hor}_{x}^{*}, \eta_{2, x} \in \wedge^{2} \mathrm{~T}_{x}^{*} S^{2}$. Let $(\cdot, \cdot)_{\text {Hor }}$ be the Mukai pairing on Hor and $(\cdot, \cdot)_{N}$ the Mukai pairing on N. Since Hor He $_{x}$ is isomorphic to $T_{\pi(x)} N$ via $\pi_{*}$, 
$\left(\pi^{*} \rho, \pi^{*} \bar{\rho}\right)_{\text {Hor }}=(\rho, \bar{\rho})_{N} \neq 0$. In view of the compactness of $N$, for sufficiently small $\epsilon$ we have

$$
\left(e^{i \epsilon \eta} \wedge \pi^{*} \rho, e^{-i \epsilon \eta} \wedge \overline{\pi^{*} \rho}\right)_{M}=\left(2 \epsilon i \eta_{2}\right) \wedge\left(e^{i \epsilon \eta_{1}} \pi^{*} \rho, e^{-i \epsilon \eta_{1}} \pi^{*} \bar{\rho}\right)_{\text {Hor }} \neq 0 .
$$

It is straightforward to check that $e^{i \in \eta} \wedge \pi^{*} \rho$ is a $d_{\pi^{*}} H^{-c l o s e d ~ f o r m . ~ T h e r e-~}$ fore $e^{i \epsilon \eta} \wedge \pi^{*} \rho$ determines a $\pi^{*} \mathrm{H}$-twisted generalized Calabi-Yau structure $\mathcal{J}^{\prime}$. Finally we note that

$$
(X-i \in d F) \cdot\left(e^{i \epsilon \eta} \wedge \pi^{*} \rho\right)=\left(i_{X}(\epsilon \eta)-i \epsilon d F\right) \wedge\left(e^{i \epsilon \eta} \wedge \pi^{*} \rho\right)=0 .
$$

This proves that the $X-i \in d F \in C^{\infty}(L)$, where $L$ is the $i$-eigenbundle of $\mathcal{J}^{\prime}$. Thus the $S^{1}$ action is Hamiltonian with the generalized moment map $\epsilon \mathrm{F}$ and trivial moment one form.

Remark 7.2. a) Topologically, $M=P \times_{S^{1}} S^{2}$ depends only on the choice of integral cohomology class $[c] \in \mathrm{H}^{2}(\mathrm{~N})$. When $[c] \neq 0$, it is easy to see that the $S^{2}$-bundle $M$ is non-trivial, i.e., not a Cartesian product of $\mathrm{N}$ an $\mathrm{S}^{2}$.

b) It is useful to have the following explicit description of $\eta$. Observe that $\mathrm{d} \theta-\Theta$ is a basic form on $(P \times \mathbb{R}) \times S^{2}$. Its restriction to the zero level of $(P \times \mathbb{R}) \times S^{2}$ descends to a one form $\gamma$ on $M$ whose restriction to each fibre $S^{2}$ is just $d \theta$. It is easy to see that on the associated bundle $P \times_{S^{1}}\left(S^{2}-\{\right.$ two poles $\left.\}\right)$ we actually have $\eta=$ $\mathrm{F} \pi_{M}^{*} \mathrm{c}+\gamma \wedge \mathrm{dF}$. Therefore, the generalized complex quotient taken at the level $\epsilon<\mathrm{t}<\epsilon$ is $M$ with the quotient generalized CalabiYau structure $e^{i t c} \wedge \rho$.

Example 7.3. Let $N=T^{4} \cong \mathbb{C}^{2} / \mathbf{Z}^{4}$ be the four dimensional torus with periodic coordinates $z_{i}=x_{i}+y_{i}, 1 \leq i \leq 2$, and let

$$
c=d x_{1} \wedge d y_{1}, \rho_{1}=e^{-i c} d z_{2}, \rho_{2}=d z_{1} \wedge d z_{2}
$$

Then it is easy to check that both $\rho_{1}$ and $\rho_{2}$ are generalized Calabi-Yau structures on $\mathrm{N}$ which are of type 1 and 2 respectively.

Let $P$ be the principal $S^{1}$-bundle $P$ with Euler class [c], $M=P \times S^{1} S^{2}$ the $S^{2}$-bundle associated to $P$, and $\rho_{i}^{\prime}:=e^{-i c} \pi^{*} \rho_{i}$ the generalized CalabiYau structures on $M$ as constructed in Theorem 7.1. By Remark 7.2 the generalized complex quotient taken at the level set $-\epsilon<t<\epsilon$ is $M$ with the quotient generalized Calabi-Yau structure $\widetilde{\rho}_{i}^{\prime}=e^{-i t c} \wedge \rho_{i}, i=1,2$. Note that $\mathrm{N}$ is endowed with the orientation $\left(\widetilde{\rho}_{i}^{\prime},{\overline{\widetilde{\rho}^{\prime}}}_{i}{ }_{i}\right)$, where $(\cdot, \cdot)$ stands for the Mukai pairing on $\mathrm{N}$. For convenience, we will denote by $\mathrm{N}^{+}$the manifold $N$ with the orientation $d x_{1} \wedge d x_{2} \wedge d x_{3} \wedge d x_{4}$, and $N^{-}$the manifold $N$ with the orientation $-d x_{1} \wedge d x_{2} \wedge d x_{3} \wedge d x_{4}$. As straightforward calculation 
shows that the Duistermaat-Heckman function for $\left(M, \rho_{1}^{\prime}\right)$ is

$$
\begin{aligned}
f_{1}(t) & =-\frac{\pi}{2} \int_{N^{+}}\left(e^{-i(t+1) c} d z_{2}, e^{i(t+1) c} d \bar{z}_{2}\right) \\
& =-\frac{\pi}{2} \int_{N^{+}} 4(t+1) d x_{1} \wedge d y_{1} \wedge d x_{2} \wedge d y_{2} \\
& =-2 \pi(t+1) ;
\end{aligned}
$$

whereas the Dustermaat-Heckman function for $\left(M, \rho_{2}^{\prime}\right)$ is

$$
\begin{aligned}
f_{2}(t) & =-\frac{\pi}{2} \int_{N^{-}}\left(e^{-i t c} \wedge d z_{1} \wedge d z_{2}, e^{i t c} \wedge d \bar{z}_{1} \wedge d \bar{z}_{2}\right) \\
& =-\frac{\pi}{2} \int_{N^{-}}-4 d x_{1} \wedge d y_{1} \wedge d x_{2} \wedge d y_{2} \\
& =-2 \pi .
\end{aligned}
$$

APPENDIX A. THE EQUIVARIANT COHOMOLOGY WITH COEFFICIENTS IN THE RING OF FORMAL POWER SERIES

In this appendix, we introduce the equivariant differential forms with coefficients in the ring of formal power series. Let an $n$ dimensional compact connected Lie group $G$ act on an $m$ dimensional manifold $M$, let $\mathfrak{g}$ be the Lie algebra of $G$. Choose a basis $\xi_{1}, \xi_{2}, \cdots, \xi_{n}$ of $\mathfrak{g}$ and let $x_{1}, x_{2}, \cdots, x_{n}$ denote the corresponding dual basis in $\mathfrak{g}^{*}$ which generate the polynomial $\operatorname{ring} S \mathfrak{g}^{*}$. Let $R=\mathbb{C}\left[\left[x_{1}, x_{2}, \cdots, x_{n}\right]\right]$ be the ring of formal power series over $\mathbb{C}$.

Definition A.1. Define

$$
\widehat{\Omega}_{\mathrm{G}}(M)=\left(\mathbb{C}\left[\left[x_{1}, x_{2}, \cdots, x_{n}\right]\right] \otimes \Omega(M)\right)^{\mathrm{G}},
$$

to be the space of equivariant differential forms with coefficients in $\mathrm{R}$, and define the equivariant differential

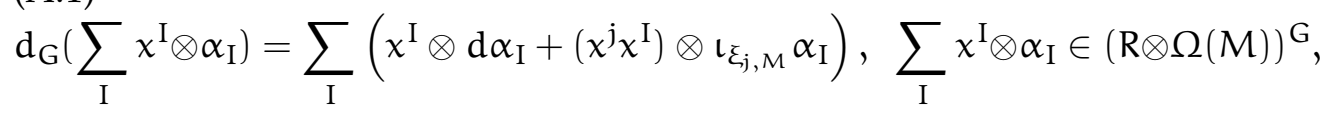

where I is a multi-index, and $\xi_{j, M}$ denotes the vector field induced by $\xi_{j} \in \mathfrak{g}$. It is easy to check that $\mathrm{d}_{\mathrm{G}}^{2}=0$. Define the cohomology

$$
\mathrm{H}\left(\widehat{\Omega}_{\mathrm{G}}(\mathrm{M}), \mathrm{d}_{\mathrm{G}}\right)=\operatorname{ker} \mathrm{d}_{\mathrm{G}} / i m \mathrm{~d}_{\mathrm{G}}
$$

to be the equivariant cohomology with coefficients in $\mathrm{R}$.

Let us give a more intrinsic description of $\widehat{\Omega}_{\mathrm{G}}(\mathrm{M})$, the space of equivariant differential forms with coefficients in $\mathbb{C}\left[\left[x_{1}, x_{2}, \cdots, x_{n}\right]\right]$. Let $a=$ $\left(x_{1}, x_{2}, \cdots, x_{n}\right)$ be the ideal in $S \mathfrak{g}^{*}=\mathbb{C}\left[x_{1}, x_{2}, \cdots, x_{n}\right]$ generated by $x_{1}, x_{2}, \cdots, x_{n}$. Then the sequence of ideals

$$
\mathrm{Sg}^{*}=\mathrm{a}^{0} \supseteq \mathrm{a} \supseteq \cdots \supseteq \mathrm{a}^{\mathrm{k}} \supseteq \cdots
$$


defines an a-adic topology on the polynomial ring $\mathrm{Sg}^{*}$, c.f. AM99, Chapter $10]$, and $R=\mathbb{C}\left[\left[x_{1}, x_{2}, \cdots, x_{n}\right]\right]=\widehat{S g^{*}}$ is the completion of $S_{\mathfrak{g}^{*}}$ under the a-adic topology. Next consider the $S_{\mathfrak{g}^{*}}$-module $\mathrm{Sg}^{*} \otimes \Omega(M)$. The sequence of modules

$$
S \mathfrak{g}^{*} \otimes \Omega(M) \supseteq \mathfrak{a}\left(\mathrm{Sg}^{*} \otimes \Omega(M)\right) \supseteq \cdots \supseteq \mathrm{a}^{\mathrm{k}}\left(\mathrm{Sg}^{*} \otimes \Omega(M)\right) \supseteq \cdots
$$

defines an a-adic topology on $\mathrm{Sg}^{*} \otimes \Omega(M)$. So $\left(\mathrm{Sg}^{*} \otimes \Omega(M)\right)^{\mathrm{G}}$ inherits a topology from $S_{\mathfrak{g}}{ }^{*} \otimes \Omega(M)$ as a subspace. Henceforth we will topologize $\left(\mathrm{Sg}^{*} \otimes \Omega(M)\right)^{\mathrm{G}}$ as a topological subspace of $\mathrm{Sg}^{*} \otimes \Omega(M)$ with the a-adic topology. Note that $\mathbb{C}\left[\left[x_{1}, x_{2}, \cdots, x_{n}\right]\right] \otimes \Omega(M)$ is the completion of $S_{\mathfrak{g}}{ }^{*} \otimes$ $\Omega(M)$ under the a-adic topology. Also note that an element $\Sigma_{I} x^{I} \otimes \alpha_{I} \in$ $\left(\mathbb{C}\left[\left[x_{1}, x_{2}, \cdots, x_{n}\right]\right] \otimes \Omega(M)\right)^{G}$ if and only if each homogenous component $\chi^{\mathrm{I}} \otimes \alpha_{\mathrm{I}} \in\left(\mathrm{Sg}^{*} \otimes \Omega(M)\right)^{\mathrm{G}}$. Thus we conclude that $\widehat{\Omega}_{\mathrm{G}}(\mathrm{M})$ is the completion of $\Omega_{\mathrm{G}}(\mathrm{M})=\left(\mathrm{Sg}^{*} \otimes \Omega(M)\right)^{\mathrm{G}}$. After these preparatory remarks, we are ready to state the following lemma.

Lemma A.2. Assume that $\mathrm{G}$ acts freely on the manifold $M$. Then the usual equivariant differential $\mathrm{d}_{\mathrm{G}}: \Omega_{\mathrm{G}} \rightarrow \Omega_{\mathrm{G}}$, the chain homotopy operator $\mathrm{Q}: \Omega_{\mathrm{G}} \rightarrow \Omega_{\mathrm{G}}$ as defined in (2.4), and the Cartan map $\mathcal{C}: \Omega_{\mathrm{G}} \rightarrow \Omega_{\text {bas }}$ as defined in (2.3) are continuous map from $\Omega_{\mathrm{G}}$ to itself.

Proof. We divide our proofs into three steps.

1) $d_{G}: \Omega_{G} \rightarrow \Omega_{G}$ is continuous.

Note that as an operator $d_{G}$ is also defined on $S \mathfrak{g}^{*} \otimes \Omega(M)$. Furthermore, we have

$$
d_{G}\left(a^{k}\left(S g^{*} \otimes \Omega(M)\right)\right) \subset a^{k}\left(S \mathfrak{g}^{*} \otimes \Omega(M)\right)
$$

for any $k \geq 0$. So $d_{G}: S \mathfrak{g}^{*} \otimes \Omega(M) \rightarrow S \mathfrak{g}^{*} \otimes \Omega(M)$ is continuous and so its restriction to $\Omega_{\mathrm{G}}$ is also continuous.

2) The chain homotopy map $Q$ is continuous.

Let $K, R$ and $F$ be the operators we introduced in Section 2.2 . Note that $\mathrm{K}, \mathrm{RF}$ and $\mathrm{Q}$ are all defined on $\mathrm{Sg}^{*} \otimes \Omega(M)$ as well, and we have

$$
\begin{gathered}
K\left(a^{k}\left(S \mathfrak{g}^{*} \otimes \Omega(M)\right)\right) \subset a^{k-1}\left(S g^{*} \otimes \Omega(M)\right), \\
(R F)^{i}\left(a^{k}\left(S \mathfrak{g}^{*} \otimes \Omega(M)\right)\right) \subset a^{k-i}\left(S \mathfrak{g}^{*} \otimes \Omega(M)\right),
\end{gathered}
$$

where $0 \leq i \leq k$. Thus $K$ and RF are both continuous operators and so their restrictions to $\Omega_{\mathrm{G}}$ are also continuous. Note that given any $\gamma \in \mathrm{Sg}^{*} \otimes \Omega(M), \mathrm{R}=\mathrm{d} \theta^{\mathrm{r}} \partial_{\mathrm{r}}$ increases the form degree of $\gamma$ by two, whereas $F$ preserves both the form degree and polynomial degree of $\gamma$. So for $i>\frac{1}{2} \operatorname{dim} M$ we have $(R F)^{i}=0$ for dimension reasons. Therefore we conclude that $\mathrm{Q}=\mathrm{KF}\left(\mathrm{RF}+(\mathrm{RF})^{2}+\cdots\right)$ is also continuous on $\mathrm{Sg}^{*} \otimes \Omega(M)$ and so the restriction of $Q$ is continuous on $\Omega_{\mathrm{G}}$. 
3) The Cartan map $\mathcal{C}: \Omega_{\mathrm{G}} \rightarrow \Omega_{\text {bas }}$ is continuous.

This follows from the simple observation that, for dimension reasons, by (2.3) we have $\mathcal{C}\left(\mathrm{a}^{\mathrm{k}}\left(\mathrm{Sg}^{*} \otimes \Omega(M)\right)=0\right.$ if $k>\frac{1}{2} \operatorname{dim} M$.

As we have explained, $\widehat{\Omega}_{\mathrm{G}}(M)$ is the completion of $\Omega_{\mathrm{G}}(M)$. Thus $\mathrm{d}_{\mathrm{G}}$, $\mathrm{Q}$, and $\mathcal{C}$ have an unique extension to $\widehat{\Omega}_{\mathrm{G}}(\mathrm{M})$. One checks easily that the extension of $\mathrm{d}_{\mathrm{G}}$ to $\widehat{\Omega}_{\mathrm{G}}$ is the one as we defined in Definition A.1, and the extension of the Cartan map $\mathcal{C}: \widehat{\Omega}_{\mathrm{G}} \rightarrow \Omega_{\text {bas }}$ is given by

$$
\sum_{\mathrm{I}} x^{\mathrm{I}} \otimes \alpha_{\mathrm{I}} \rightarrow \sum_{\mathrm{I}} c^{\mathrm{I}} \wedge \alpha_{\mathrm{I}}
$$

where $c^{i}$ is the curvature elements as defined in (2.2), and $I=\left(i_{1}, i_{2}, \cdots, i_{n}\right)$ is a multi-index. Note that if $|I|=\sum_{k=1}^{n} i_{k}>\frac{1}{2} \operatorname{dim} M$, then $c^{I} \wedge \alpha_{I}=0$ for dimension reasons. So the right hand side of (A.2) is actually a finite sum. Since the equality $\mathrm{d}_{\mathrm{G}} \mathrm{Q}+\mathrm{Qd} \mathrm{d}_{\mathrm{G}}=\mathrm{I}-\mathcal{C}$ holds on $\Omega_{\mathrm{G}}$, it also holds on the completion of $\Omega_{\mathrm{G}}$, i.e., $\widehat{\Omega}_{\mathrm{G}}$.

In summary, we have proved the following result.

Theorem A.3. Assume the action of $\mathrm{G}$ on $\mathrm{M}$ is free. Then the Cartan map (A.2) induces a natural isomorphism from $\mathrm{H}\left(\widehat{\Omega}_{\mathrm{G}}(\mathrm{M}), \mathrm{d}_{\mathrm{G}}\right)$ to the ordinary cohomology $\mathrm{H}(\mathrm{M} / \mathrm{G})$.

Indeed, we can prove a slightly more general result. Suppose that $H \in$ $\Omega^{3}(M)$ is a closed basic three form. Then the map

$$
\mathrm{H} \wedge: \Omega_{\mathrm{G}} \rightarrow \Omega_{\mathrm{G}}, \quad \alpha \mapsto \mathrm{H} \wedge \alpha
$$

is obviously continuous. Since $\mathrm{H}$ is a basic form, a straightforward check shows that $\mathrm{Q}(\mathrm{H} \wedge \alpha)+\mathrm{H} \wedge(\mathrm{Q} \alpha)=0$ for any $\alpha \in \Omega_{\mathrm{G}}$. As a result, $\mathrm{d}_{\mathrm{G}, \mathrm{H}} \mathrm{Q}+$ $\mathrm{Qd}_{\mathrm{G}, \mathrm{H}}=\mathrm{I}-\mathcal{C}$ holds on $\Omega_{\mathrm{G}}$, and so it also holds on $\widehat{\Omega}_{\mathrm{G}}$. This proves the following result.

Theorem A.4. Assume the action of $\mathrm{G}$ on $\mathrm{M}$ is free. Let $\pi: M \rightarrow M / G$ be the quotient map. And assume that $\mathrm{H} \in \Omega^{3}(\mathrm{X})$ is a basic form so that there is a three form $\widetilde{\mathrm{H}} \in \Omega^{3}(\mathrm{X} / \mathrm{G})$ which satisfies $\pi^{*} \widetilde{\mathrm{H}}=\mathrm{H}$. Then the Cartan map (A.2) induces a natural isomorphism from $\mathrm{H}_{\mathrm{G}}(\mathrm{M}, \mathrm{H})$ to the twisted cohomology $\mathrm{H}(\mathrm{M} / \mathrm{G}, \widetilde{\mathrm{H}})$.

\section{REFERENCES}

[AS05] M. Atiyah, G. Segal, Twisted K-theory and cohomology, preprint, math.KT/0510674.

[AM99] M. Atiyah, I.G. Macdonald, Introduction to commutative algebra, Addison-Wesley Publishing Co.; Reading, Mass-London-Don Mills, Ont. 1969.

[BCRR05] U. Bruzzo, L. Cirio, P. Rossi, and V. Rubtsov, Equivariant cohomology and locolization for Lie algebroids, mah.DG/0506392

[BCG05] H. Bursztyn, G. Cavalcanti, and M. Gualtieri, Reduction of Courant algebroids and generalized complex structures, math.DG/0509640. Adv. in Math., 211 (2), 726 - 765, 2007.

[Ca05] Gil, Cavalcanti, New aspects of $\mathrm{dd}^{\mathrm{c}}$ lemma, Oxford D. Phil. thesis, math.DG/0501406 
[Ca06] Gil, Cavalcanti, The decomposition of forms and cohomology of generalized complex manifolds, J. Geom. Phyi. 57, 121-132, 2006

[Che97] C. Chevalley, The algebraic theory of spinors and Clifford algebras, Collected works, VOl. 2., Edited and with a forward by Pierre Cartier and Catherine Chevalley. With a postface by J.-P. Bourguignon. Springer-Verlag, Berlin, 1997. xiv+214 pp.

[GHR84] Jr. S. Gates, C. Hull, and M. Rocek, Twisted multiplets and new supersymmetric nonlinear 6-model, Nuclear Phys. B. 248(1) 157- 186, 1984.

[Gua03] M. Gualtieri, Generalized complex geometry, Oxford D. Phil. thesis, math.DG/0401221

[Gua07] M. Gualtieri, Generalized complex geometry, math.DG/0703298

[Gua04] Marco Gualtieri Generalized geometry and the Hodge decomposition, math.DG/0409093

[GS84] V. Guillemin, S.Sternberg, Symplectic Techniques in Physics, Cambridge University Press, Cambridge, 1984.

[GS99] V. Guillemin, S.Sternberg, Super-symmetry and equivariant de Rham theory, With an appendix containing two reprints by Henri Cartan. Mathematics Past and Present. Springer-Verlag, Berlin, 1999.

[GGK97] V. Guillemin, V. Ginzburg, Y. Karshon, Moment maps, Cobordisms, and Hamiltonian actions, American Mathematical Society, Mathematical surveys and Monographys, Vol. 98.

[H02] N. Hitchin, Generalized Calabi-Yau manifolds, Q.J. Math., 54 (3) 281-308, 2003

[Hu05] S. Hu, Hamiltonian symmetries and reduction in generalized geometry, math.DG/0509060

[HuUribe06] S. Hu, B. Uribe, Extended manifolds and extended equivariant cohomology, math.DG/0608319

[Kir86] F., Kirwan, Cohomology of quotients in symplectic and algebraic geometry, Mathematical Notes, 31, Princeton, NJ, 1984.

[KL04] A. Kapustin, Y. Li, Topological sigma-models with H-flux and twisted generalized complex manifolds, hep-th/0407249

[KT06] A. Kapustin, A. Tomasiello, The general $(2,2)$ gauged sigma model with three-form flux, preprint, hep-th/0610210.

[LS03] Yi Lin, Reyer Sjamaar, Equivariant symplectic Hodge theory and the $\mathrm{d}_{\mathrm{G}} \delta$-lemma, J. Symplectic Geometry, 2 (2004), no. 2, 267-278.

[L04] Yi Lin, Examples of non-Kähler Hamiltonian circle manifolds with the strong Lefschetz property, Adv. in Math., 208 (207), issue 2, 699-709.

[LT05] Yi Lin, Susan Tolman, Symmetries in generalized Kähler geometry, Comm. Math. Phys., 208 (2006), 199-222, math.DG/0509069

[Lin06] Yi Lin, Generalized geometry, equivariant $\bar{\partial}$-lemma and torus actions, the Journa of geometry and physics, 57 (2007) 1842-1860, also available on arxiv: math.DG/0607401

[Mc01] J. McCleary, A user's Guide to Spectral Sequences, Second edition. Cambridge Studies in Advanced Mathematics, 58. Cambridge University press, Cambridge, 2001.

[NY06] Yasufumi Nitta, Reduction of generalized Calabi-Yau structures, to appear, J. Math. Soc. Japan, Vol 59, no. 4 (2007), also available on arxiv: math.DG/0611341

[NY07] Yasufumi Nitta, Duistermaat-Heckman formula for a Torus action on a generalized Calabi-Yau manifold and localization formula, math.DG/0702264

[SX05] M. Stiénon and X. Ping, Reduction of generalized complex structure, math.DG/0509393

[Va05] Vaisman, I. Reduction and submanifolds of generalized complex manifolds, math.DG/0511013

[Yan96] D. Yan, Hodge structure on symplectic manifolds, Adv. in Math. 120 (1996), no. 1, 143154. 